\title{
A polarity pathway for exocyst-dependent intracellular tube extension
}

\author{
Joshua Abrams ${ }^{1}$ and Jeremy Nance ${ }^{1,2, *}$
}

1Skirball Institute of Biomolecular Medicine, NYU Grossman School of Medicine, New York, NY 10016

${ }^{2}$ Department of Cell Biology, NYU School of Medicine, New York, NY 10016

${ }^{*}$ Correspondence: Jeremy Nance

NYU School of Medicine

Skirball Institute of Biomolecular Medicine

540 First Avenue, $4^{\text {th }}$ floor lab 17

New York, NY 10016

212-263-3156

Jeremy.Nance@med.nyu.edu 


\section{ABSTRACT}

Lumen extension in intracellular tubes can occur by the directed fusion of vesicles with an invading apical membrane domain. Within the C. elegans excretory cell, which contains an intracellular tube, the exocyst vesicle-tethering complex is enriched at the lumenal membrane domain and is required for tube formation, suggesting that it targets vesicles needed for lumen extension. Here, we identify a polarity pathway that promotes intracellular tube formation by enriching the exocyst at the lumenal membrane. We show that the PAR polarity proteins PAR-6 and PKC-3/aPKC localize to the lumenal membrane domain and function within the excretory cell to promote lumen extension, similar to exocyst component SEC-5 and exocyst regulator RAL-1. Using acute protein depletion, we find that PAR-6 is required to recruit the exocyst to the lumenal membrane domain, whereas PAR-3, which functions as an exocyst receptor in mammalian cells, appears to be dispensable for exocyst localization and lumen extension. Finally, we show that the Rho GTPase CDC-42 and the RhoGEF EXC5/FGD act as upstream regulators of lumen formation by recruiting PAR-6 and PKC-3 to the lumenal membrane. Our findings reveal a molecular pathway that connects Rho GTPase signaling, cell polarity, and vesicle-tethering proteins to promote lumen extension in intracellular tubes. 


\section{INTRODUCTION}

Most organs contain tubes, which are used to transport gases and fluids from one site within the body to another. The circumference of larger tubes, such as the human intestine, is lined by many cells connected to one another with junctions. By contrast, the smallest tubes have intracellular lumens that are contained entirely within the cytoplasm of a cell. Although some intracellular tubes arise when a cell wraps circumferentially and recontacts itself to hollow out a lumen from the extracellular space (Rasmussen et al., 2008; Stone et al., 2009), many intracellular tubes are thought to form when an apical membrane domain invades into the cytoplasm to become the lumen (Lubarsky and Krasnow, 2003; Sundaram and Cohen, 2017). The C. elegans excretory cell provides a powerful model system for studying this mechanism of intracellular lumen formation. Born during late embryogenesis, the $\mathrm{H}$-shaped excretory cell contains four long canal arms that grow during larval stages to extend nearly the full length of the worm, terminating growth during the final L4 larval stage (Nelson et al., 1983). An intracellular lumen initiates within the cell body and invades the length of each canal arm, functioning in osmoregulation (Buechner et al., 1999; Mancuso et al., 2012; Nelson and Riddle, 1984; Sundaram and Buechner, 2016). Vertebrate capillaries, as well as terminal and fusion cells of the Drosophila trachea and the Ciona notochord, are additional examples of cells containing intracellular tubes that are thought to form through an apical invasion mechanism (Denker et al., 2013; Gervais and Casanova, 2010; Herwig et al., 2011; Lenard et al., 2013). 
Formation of an intracellular lumen by apical domain invasion requires the polarized delivery and fusion of vesicles, which supply the new membrane needed to extend the lumenal surface (Berry et al., 2003; Gervais and Casanova, 2010; Khan et al., 2013; Kolotuev et al., 2013; Schottenfeld-Roames and Ghabrial, 2012). The highly conserved, eight-protein exocyst complex and the small GTPase exocyst activator Ral are required for polarized membrane targeting of vesicles in many cell types (Wu and Guo, 2015). The exocyst mediates vesicle tethering and subsequent fusion at sites where it enriches on the cell membrane (He and Guo, 2009; Lipschutz et al., 2000; Liu and Guo, 2012). Studies in both yeast and mammalian cells suggest that the eight exocyst subunits (Sec3, Sec5, Sec6, Sec8, Sec10, Sec15, Exo70, and Exo84) assemble together from distinct subcomplexes to promote vesicle tethering (Ahmed et al., 2018; Heider et al., 2016). Active Ral GTPase binds directly to the exocyst to promote its assembly (Brymora et al., 2001; Chen et al., 2011; Moskalenko et al., 2002; Moskalenko et al., 2003; Sugihara et al., 2002). The exocyst is enriched at the lumenal membrane of Drosophila and C. elegans intracellular tubes and is required for proper lumenogenesis (Armenti et al., 2014a; Jones et al., 2014), suggesting that it targets the vesicles needed for lumen extension. A key unanswered question is how exocyst localization becomes polarized to accumulate on the lumenal membrane.

PAR proteins, which include Par3 (a multi-PDZ domain scaffolding protein), Par6 (a PDZ and CRIB domain scaffolding protein), and aPKC (atypical protein kinase C), mediate cell polarity by establishing an asymmetric signaling domain at the plasma membrane (Nance and Zallen, 2011; St Johnston and Ahringer, 2010). Upstream 
polarity cues can induce PAR asymmetries by activating the Rho GTPase Cdc42, which binds directly to the Par6 CRIB domain, recruiting Par6 and its binding partner aPKC to the membrane and promoting aPKC kinase activity (Aceto et al., 2006; Gotta et al., 2001; Hutterer et al., 2004; Joberty et al., 2000; Johansson et al., 2000; Kay and Hunter, 2001; Lin et al., 2000; Qiu et al., 2000). Par6 and aPKC are also concentrated within asymmetric membrane domains by interacting with Par3 (Tabuse et al., 1998; Watts et al., 1996). PAR proteins regulate downstream effectors through aPKC phosphorylation or by recruiting effector proteins directly (Nance and Zallen, 2011; St Johnston and Ahringer, 2010).

PAR proteins are important for lumen formation in both multicellular and intracellular tubes. For example, in MDCK multicellular cysts grown in 3D culture, Par3 localizes to the membrane of the lumen that forms at the center of the cell cyst, and its knockdown leads to the formation of multiple, disorganized lumens (Bryant et al., 2010). In Drosophila terminal tracheal cells, Par-6 and aPKC are found at the lumenal membrane and are thought to be required for lumenogenesis (Jones and Metzstein, 2011). Within the C. elegans excretory cell, fluorescently tagged PAR-3 and PAR-6 expressed from transgenes, and endogenous PAR- 6 and PKC-3/aPKC detected by immunostaining, accumulate at the lumenal membrane (Armenti et al., 2014a). Transgenic CDC-42 and a putative activator, the RhoGEF EXC-5/FGD, are also enriched at the lumenal membrane (Lant et al., 2015; Mattingly and Buechner, 2011; Suzuki et al., 2001). Whereas exc-5 mutants have severely truncated excretory cell canals (Buechner et al., 1999; Gao et al., 2001; Suzuki et al., 2001), the contribution that PAR proteins and 
CDC-42 make to excretory cell lumenogenesis has not been fully determined because these proteins have earlier essential developmental functions (Gotta et al., 2001; Kay and Hunter, 2001; Kemphues et al., 1988; Tabuse et al., 1998; Watts et al., 1996).

Several PAR proteins have been shown to physically interact with the exocyst (Ahmed and Macara, 2017; Das et al., 2014; Lalli, 2009; Rosse et al., 2009; Zuo et al., 2011; Zuo et al., 2009), raising the possibility that PAR proteins might function in lumenogenesis by recruiting the exocyst to the lumenal membrane. In mammary epithelial cells, a lysine-rich domain of Par3 binds directly to the exocyst protein Exo70 and is thought to function as an exocyst receptor, recruiting the complex to sites where Par3 is enriched (Ahmed and Macara, 2017). Within migrating rat kidney epithelial cells, aPKC interacts with the exocyst through the aPKC-binding protein Kibra and is required for exocyst enrichment at the leading edge, although exocyst is also required for aPKC localization to this site (Rosse et al., 2009). In mammalian neurons, the PDZ domain of Par6 can bind the exocyst (through Ex084), and this interaction requires active Ral GTPase (Das et al., 2014). These observations raise the possibility that Par3, Par6 and/or aPKC are required to enrich the exocyst at the lumenal membrane during intracellular tubulogenesis. Consistent with this model, Sec8 enrichment at the lumenal membrane domain in aPKC mutant Drosophila terminal tracheal cells is lost (Jones et al., 2014). However, the lumen and branching defects of aPKC mutant tracheal cells make it difficult to establish whether aPKC recruits the exocyst directly to the lumenal membrane, or whether exocyst loss from the lumenal membrane arises indirectly as a result of other aPKC-dependent cellular defects. Testing whether PAR proteins recruit 
the exocyst during intracellular tube formation would ideally be accomplished by eliminating PAR proteins acutely, after lumenogenesis is complete, and determining if exocyst localization is altered.

Here, we utilize degron-tagged alleles of SEC-5, RAL-1, PAR-3, PAR-6, PKC-3, CDC42, and EXC-5 to establish the roles of these proteins in extending the excretory cell intracellular lumen. We show that PAR-6 and PKC-3, but not PAR-3, are essential for lumen extension, and using acute protein depletion we demonstrate that PAR-6, but not PAR-3, is needed to recruit the exocyst to the lumenal membrane. Finally, we provide evidence that EXC-5 and CDC-42 function upstream of PAR-6 and PKC-3 as polarity cues, recruiting these proteins to the lumenal membrane. Our findings identify a pathway that connects Rho GTPase, cell polarity, and vesicle-tethering proteins to lumen extension during intracellular tubulogenesis.

\section{RESULTS}

SEC-5 and RAL-1 function within the excretory cell to promote lumen extension The enrichment of the exocyst at the excretory cell lumenal membrane and its requirement for proper lumenogenesis suggest that exocyst-dependent vesicle delivery provides the new membrane needed for lumen expansion (Armenti et al., 2014a). If so, the exocyst, which is broadly expressed and needed for embryonic development (Armenti et al., 2014a; Frische et al., 2007), should be required autonomously within the excretory cell. To test this hypothesis, we designed a degron-based strategy to conditionally deplete exocyst component SEC-5 and exocyst activator RAL-1 (the sole 
C. elegans Ral GTPase homologue) specifically within the excretory cell (Figure 1A); this approach removes zygotically expressed protein as well as inherited maternal protein, which can otherwise mask mutant phenotypes (Nance and Frokjaer-Jensen, 2019). Proteins tagged with the ZF1 degron are rapidly degraded to undetectable levels by expressing the E3 ubiquitin ligase substrate-adapter protein ZIF-1 (Armenti et al., 2014b; DeRenzo et al., 2003; Reese et al., 2000). In order to express ZIF-1 specifically within the excretory cell, we searched for an excretory cell-specific promoter. Existing transcriptional reporters for two promoters described to be active predominantly or exclusively in the excretory cell, pgp-12 (Zhao et al., 2005) and glt-3 (Mano et al., 2007), showed additional expression in other embryonic tissues. Using the WormBase (www.wormbase.org) data-mining platform WormMine, we identified additional candidate promoters among a set of genes described to be expressed specifically within the excretory cell. Upstream sequences of one gene, $T 28 H 11.8$, drove detectable mCherry expression specifically in the excretory cell from embryogenesis onward (Figure S1). To determine if ZIF-1 expressed from the T28H11.8 promoter (hereafter excP) was sufficient to degrade ZF1-tagged proteins specifically within the excretory cell, we introduced a high-copy array containing excP::zif-1 into worms expressing a ZF1-tagged reporter protein, ZF1::GFP::CDC-42. Control larvae, which did not inherit the excP::zif-1 array, robustly expressed ZF1::GFP::CDC-42 in the excretory cell and other tissues (Figure 1B). By contrast, ZF1::GFP::CDC-42 was depleted below detectable levels within the excretory cell in larvae that inherited the excP::zif-1 transgenic array (Figure 1C), whereas expression of ZF1::GFP::CDC-42 persisted in 
other tissues. We conclude that excP::zif-1 can be used to deplete ZF1-tagged proteins from the excretory cell.

In order to inhibit exocyst activity specifically within the excretory cell, we created a high-copy, integrated excP::zif-1 transgene to conditionally degrade ZF1-tagged SEC-5 and RAL-1 proteins. For SEC-5, we utilized sec-5(xn51), a functional, endogenously tagged sec-5::zf1::yfp allele (Armenti et al., 2014b). Similar to SEC-5::YFP protein expressed from a transgene (Armenti et al., 2014a), endogenously tagged SEC$5:: Z F 1:: Y F P$ concentrated at the excretory cell lumenal membrane (Figure 1D). For RAL-1, we utilized the ral-1(tm5205) null mutation rescued by a previously characterized, low-copy, functional ral-1P::zf1::yfp::ral-1 transgene (Armenti et al., 2014a). We examined phenotypes of worms with excretory cell-specific depletion of SEC-5::ZF1::YFP (SEC-5 ${ }^{\text {exc(-) }}$ worms) or ZF1::YFP::RAL-1 (RAL-1 ${ }^{\text {exc(-) }}$ worms) using coexpressed markers of the excretory cell cytoplasm (excP::yfp) and lumenal membrane (ifb-1::cfp) (see Figure 1A). Controls expressing excP::zif-1 but not the ZF1-tagged proteins displayed normal excretory canal outgrowth and morphology (Figure 1E,F-F”). In contrast to controls, SEC-5 ${ }^{\mathrm{exc}(-)}$ and $\mathrm{RAL}-1^{\mathrm{exc}(-)}$ larvae had severely truncated, swollen canals with disorganized, cystic lumens (Figure 1G-J"). We measured canal length by examining where the posterior canal lumens ended relative to body length in L1 and L4 larvae, as these stages represent active outgrowth (L1) and maintenance (L4) of the canal lumen. Dividing the body into quartiles along its anterior-posterior axis, nearly all control larvae extended canals to the third quartile (51-75\% of body length) at the L1 stage and the fourth quartile (76-100\% of body length) by the L4 stage (Figure 2). 


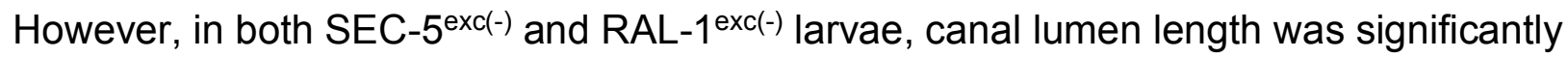
reduced at both L1 and L4 stages, with nearly all larvae containing canal lumens that extended to less than $50 \%$ body length (Figure 2). The canal lumen length defect of

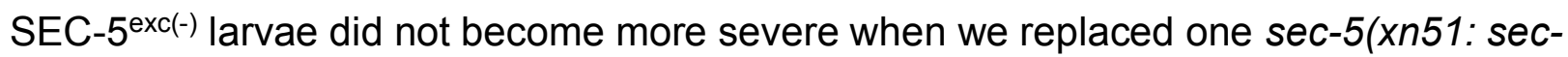
$5:: z f 1:: y f p)$ allele with the sec-5(tm1443) predicted null allele (Frische et al., 2007) (Figure S2), suggesting that SEC-5 ${ }^{\text {exc(-) }}$ phenotypes result from nearly complete or complete loss of SEC- 5 protein. Together, these data indicate that exocyst activity within the excretory cell is needed for proper organization and extension of its intracellular lumen.

PAR proteins and CDC-42 are expressed in the excretory cell and have distinct localization patterns

We next addressed whether PAR proteins are required for extension of the excretory cell lumen using endogenously tagged alleles of par-3, par-6, and pkc-3 expressing fusion proteins tagged with ZF1 and either YFP or GFP. par-3::zf1::yfp (this study), par6::zf1::yfp (Zilberman et al., 2017) and zf1::gfp::pkc-3 (Montoyo-Rosario et al., 2020) knock-in alleles were functional, as they did not cause the embryonic lethality (Kemphues et al., 1988; Tabuse et al., 1998; Watts et al., 1996) associated with par-3, par-6, or pkc-3 inactivation (see Materials and Methods) (Montoyo-Rosario et al., 2020; Zilberman et al., 2017). PAR-3::ZF1::YFP, PAR-6::ZF1::YFP and ZF1::GFP::PKC-3 proteins each concentrated at the excretory cell lumenal membrane within puncta (Figure 3A-C), similar to SEC-5::ZF1::YFP (Figure 1D). 
The localization of CDC-42 within the excretory cell has only been described using highcopy transgenes and heterologous promoters (Lant et al., 2015; Mattingly and Buechner, 2011), and the high-copy transgene expressing ZF1::GFP::CDC-42 that we used to test the efficacy of excP::zif-1 (Figure 1B) (Armenti et al., 2014b). We examined CDC-42 subcellular localization in the excretory cell using a functional endogenously tagged zf1::yfp::cdc-42 allele (Zilberman et al., 2017). ZF1::YFP::CDC-42 protein was present at the lumenal membrane (Figure 3G). However, ZF1::YFP::CDC-42 also extended well into the excretory cell cytoplasm compared to endogenously expressed PAR-6::mKate present within the same animal (Figure 3D,G,H), whereas endogenously tagged PAR-6::ZF1::YFP and PAR-3::mCherry showed a similar enrichment to the lumenal membrane (Figure 3E-F). Thus, consistent with previous findings made using immunostaining and transgenes (Armenti et al., 2014a), endogenously tagged PAR-3, PAR-6, PKC-3 and CDC-42 are each expressed within the excretory cell and are present at the lumenal membrane, although CDC-42 is also found at high levels within the cytoplasm.

PAR-6, PKC-3 and CDC-42 are required in the excretory cell for lumen extension To determine if PAR proteins and CDC-42 are required within the excretory cell for lumenogenesis, we crossed excP::zif-1 with each par or cdc-42 knock-in allele and examined excretory canal morphology using cytoplasmic and lumenal markers (see

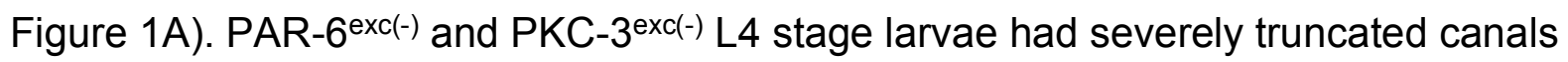

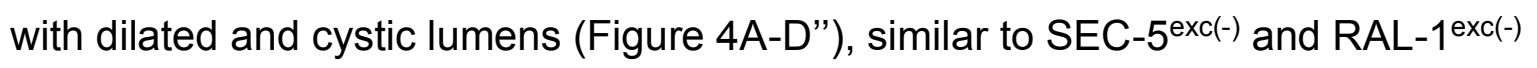
larvae (see Figure 1G-J"). CDC-42 ${ }^{\mathrm{exc}(-)}$ larvae showed similar lumen extension defects 
(Figure 4E-F'), but in addition some animals had a split-canal phenotype whereby two lumenized canals split from a single canal arm ( $n=42 / 158$ L4 larvae, Figure S3).

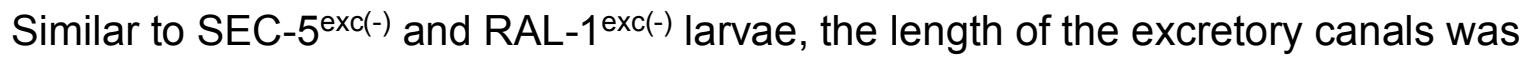
significantly shorter in PAR-6 ${ }^{\text {exc(-) }}$ PKC-3 $3^{\operatorname{exc}(-)}$, and CDC-42 ${ }^{\text {exc(-) }}$ compared to controls at both the L1 and L4 stages (Figure 2). Unexpectedly, PAR-3exc(-) larvae had a distinct and comparatively mild phenotype. At the L1 stage, canal lumens in PAR-3 $3^{\text {exc(-) larvae }}$ had an irregular diameter (Figure S4), and were significantly shorter than controls

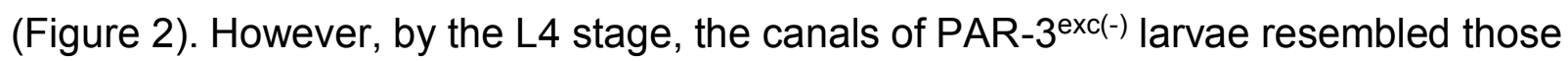
of controls (Figure 4G-H”) and were not significantly shorter (Figure 2). We conclude that PAR-6, PKC-3 and CDC-42 function within the excretory cell to promote extension of the lumen, and that PAR-3 is likely only important for lumen outgrowth during early stages, although we cannot exclude the possibility that an undescribed isoform of par-3 with a different 3' end, and thus lacking the ZF1 tag, is expressed within the excretory cell. Our findings also show that, in addition to promoting lumen extension, CDC-42 functions to prevent canal arms from bifurcating.

PAR-6, but not PAR-3, is required for exocyst lumenal membrane localization The results above suggest that exocyst function or localization may require PAR-6, PKC-3, and CDC-42, but not PAR-3. To determine if PAR proteins regulate lumen extension by recruiting exocyst to the lumenal membrane, we acutely degraded PAR$6:: Z F 1:: Y F P$ and PAR-3::ZF1::YFP protein at the L4 larval stage, after canal growth was complete, by expressing ZIF-1 from a heat-shock promoter. This approach allowed us to analyze exocyst localization in anatomically normal canals, immediately after rapid 
PAR protein depletion (Figure 5A). Following a 30-minute heat shock to induce ZIF-1 expression at the L4 stage, PAR-6::ZF1::YFP degraded rapidly within 1 hour (Figure 5B-C, Figure S5). To monitor exocyst localization after PAR-6::ZF1::YFP depletion, we utilized a transgene expressing mCherry::SEC-10 (Armenti et al., 2014a), which like SEC-5::ZF1::YFP enriches at the lumenal membrane (Figure 5B',D). After PAR$6:: Z F 1:: Y F P$ degraded, mCherry::SEC-10 was no longer enriched at the lumenal membrane, but instead, appeared evenly distributed throughout the cytoplasm (Figure $\left.5 C^{\prime}, E\right)$. We quantified these changes in localization by comparing mCherry::SEC-10 intensity along the lumenal membrane to that within the adjacent cytoplasm by generating a lumen/cytoplasm intensity ratio (Figure $5 \mathrm{~A}$ ), which was significantly reduced in PAR-6-depleted larvae (Figure 5F). We performed analogous experiments to determine the role of PAR-3 in exocyst localization. In contrast to PAR-6::ZF1::YFP depletion, loss of PAR-3::ZF1::YFP did not decrease the enrichment of mCherry::SEC10 at the lumenal membrane, despite a lack of visible PAR-3::ZF1::YFP protein following ZIF-1 induction (Figure 5G-K). We conclude that PAR-6 is required to enrich the exocyst complex at the lumenal membrane, whereas PAR-3 is likely dispensable for exocyst lumenal membrane enrichment.

\section{PAR-3 promotes PAR-6 lumenal membrane localization}

In many polarized cell types, PAR-3 helps enrich PAR-6 at the membrane (Nance and Zallen, 2011; St Johnston and Ahringer, 2010). Therefore, the requirement for PAR-6, but not PAR-3, in mCherry::SEC-10 lumenal membrane enrichment was surprising. To investigate the epistatic relationship between PAR-3 and PAR- 6 within the excretory 
cell, we first expressed ZIF-1 from a heat shock promoter and degraded PAR-

3::ZF1::YFP after canal growth was complete (Figure 6A-B). Surprisingly, endogenously tagged PAR-6::mKate (Dickinson et al., 2017) was significantly less enriched at the lumenal membrane and increased within the cytoplasm after depletion of PAR-

3::ZF1::YFP when compared to control larvae (Figure 6A'-E), although some puncta of PAR-6::mKate remained at the lumenal membrane (Figure 6B', arrowheads). In reciprocal experiments, we degraded PAR-6::ZF1::YFP by expressing ZIF-1 from a heat shock promoter and examined endogenously tagged PAR-3::mCherry localization. PAR-3::mCherry remained enriched at the lumenal membrane in PAR-6-depleted L4 worms, and unexpectedly, its lumen/cytoplasm ratio was significantly increased (Figure 6F-J). We propose that PAR-3 is required to recruit most PAR-6 to the lumenal membrane, but that the PAR-6 puncta remaining after PAR-3::ZF1::YFP depletion are sufficient to recruit the exocyst to the lumenal membrane (see Discussion). In addition, these findings show that PAR-6 limits PAR-3 lumenal membrane enrichment.

\section{$C D C-42$ is required for PAR-6 lumenal membrane localization}

We next asked what other factors act upstream to regulate the lumenal membrane enrichment of PAR-6 and PKC-3 within the excretory cell. One candidate is CDC-42, which binds to the PAR-6 CRIB domain and can recruit PAR-6 to the membrane in parallel to PAR-3 in the one-cell C. elegans embryo (Aceto et al., 2006; Beers and Kemphues, 2006; Gotta et al., 2001; Joberty et al., 2000; Kay and Hunter, 2001; Rodriguez et al., 2017; Wang et al., 2017). CDC-42 ${ }^{\text {exc(-) }}$ and PAR-6 ${ }^{\text {exc(-) }}$ larvae displayed a similar canal outgrowth phenotype (Figure 2), consistent with these two proteins 
acting in the same lumen extension pathway within the excretory cell. To determine if CDC-42 is required for PAR-6 enrichment at the lumenal membrane, we acutely degraded ZF1::YFP::CDC-42 by heat shock expression of ZIF-1 in L4 larvae. PAR6::mKate lumenal membrane enrichment was significantly decreased after loss of CDC42 (Figure 7A-E). Together, these results suggest that CDC-42 promotes lumen extension by helping to enrich PAR-6 at the lumenal membrane.

EXC-5, a putative CDC-42 RhoGEF, is required for PKC-3 lumenal membrane localization

Given that only active GTP-bound CDC-42 interacts with PAR-6 (Aceto et al., 2006; Gotta et al., 2001), we hypothesized that CDC-42 at the lumenal membrane is activated by one or more RhoGEFs. EXC-5 is an orthologue of the faciogenital dysplasiaassociated (FGD) family of RhoGEFs that can activate Cdc42 in biochemical and cell culture assays (Hayakawa et al., 2008; Huber et al., 2008; Kurogane et al., 2012; Miyamoto et al., 2003; Steenblock et al., 2014; Umikawa et al., 1999; Zheng et al., 1996), and EXC-5 has been proposed as an activator of CDC-42 in the excretory cell. C. elegans EXC-5::GFP over-expressed from a high-copy transgene is present within the excretory cell (Mattingly and Buechner, 2011; Suzuki et al., 2001), and exc-5 mutants have shortened excretory cell canals. In addition, genetic epistasis experiments are consistent with $c d c-42$ functioning downstream of exc-5 (Mattingly and Buechner, 2011; Shaye and Greenwald, 2016). To determine whether EXC-5 is required for PAR-6 or PKC-3 protein localization, as is CDC- 42 , we created an endogenously tagged exc-5 allele expressing EXC-5::ZF1::mScarlet. Like PAR-6 and PKC-3, EXC-5::ZF1::mScarlet 
was enriched at the lumenal membrane (Figure 7F-F',H). We used heat-shock inducible ZIF-1 to remove EXC-5::ZF1::mScarlet acutely and examined the effect on endogenously tagged GFP::PKC-3 (Rodriguez et al., 2017; Wang et al., 2017). Upon depletion of EXC-5::ZF1::mScarlet, GFP::PKC-3 enrichment at the lumenal membrane was significantly reduced compared to control larvae (Figure 7G-J). These results indicate that EXC-5 is required for PKC-3 recruitment to the excretory cell lumenal membrane, most likely through its activation of CDC-42.

\section{DISCUSSION}

An intracellular lumenogenesis pathway bridging Rho GTPase, cell polarization and vesicle-tethering proteins

During tubulogenesis within the $C$. elegans excretory cell, it has been proposed that the docking and subsequent fusion of large 'canalicular' vesicles at the lumenal membrane domain provides the membrane needed for tube extension (Khan et al., 2013; Kolotuev et al., 2013). We showed previously that exocyst complex activity is required for canalicular vesicles to connect with the lumenal membrane domain and for normal lumen extension to occur (Armenti et al., 2014a). Here, based on cell-specific protein depletion experiments during lumen extension, and protein localization analysis following acute protein degradation in fully developed excretory cells, we propose a pathway for lumen extension (Figure 7K). Most upstream, RhoGEF EXC-5 at the lumenal membrane activates the Rho GTPase CDC-42. Although EXC-5 has been proposed previously as an activator of CDC-42 at the lumenal membrane (Mattingly and Buechner, 2011; Shaye and Greenwald, 2016), our findings show for the first time that 
its depletion causes a similar molecular defect as depletion of CDC-42 (loss of PKC-3 or PAR-6 from the lumenal membrane). Downstream of EXC-5, we propose that active CDC-42 recruits PAR-6 and PKC-3 through interactions with the PAR-6 CRIB domain. In turn, PAR-6 and PKC-3 function together to recruit the exocyst. RAL-1 has previously been shown to promote exocyst membrane localization, including in the early $C$. elegans embryo (Armenti et al., 2014a). The comparable phenotypes we observe in SEC-5 ${ }^{\text {exc- }}$ and RAL-1 ${ }^{\text {exc- }}$ larvae suggest that it has a similar function within the excretory cell.

Although we found that in PAR-3-depleted larvae most PAR-6 was lost from the excretory cell lumenal membrane - a phenotype that could be predicted based on previous studies of PAR-3 in other cell types - the relatively mild lumen extension phenotype of PAR-3 ${ }^{\text {exc- }}$ larvae (shortened canals in the L1 stage that recovered to normal length by the L4 stage) and lack of requirement for PAR-3 in mCherry::SEC-10 localization were somewhat surprising. Although we cannot exclude the possibility that an alternative form of PAR-3 protein lacking the ZF1 degron is produced, we consider this unlikely, as no such isoforms have been described, and the loss of PAR-6 at the lumenal membrane suggests that PAR-3 depletion was effective. Instead, we favor the hypothesis that PAR-3 makes lumen extension more efficient by promoting PAR-6 lumenal enrichment, and that partial PAR-6 recruitment by CDC-42 is sufficient for lumen extension. Studies in the zygote have shown that in addition to localizing PAR-6 and PKC-3 to the membrane, CDC-42 also promotes PKC-3 activity (Rodriguez et al., 2017), raising the possibility that it plays a more consequential role during lumen 
extension than PAR-3 by both localizing and activating the PAR-6/PKC-3 complex. Such a relationship between PAR-3 and CDC-42 in recruiting PAR-6 likely occurs in additional cell types, as PAR-3 depletion in the epidermis causes PAR-6 mislocalization but not the junction defects that occur following PAR-6 depletion in the same cells (Achilleos et al., 2010). While it is not yet clear why PAR-3 appears to be more important for lumen extension at earlier larval stages, this is when active lumen outgrowth occurs. A reasonable hypothesis is that partially compromised PAR-6 function (because of reduced enrichment at the lumenal membrane) may be more consequential at this stage of lumenogenesis.

par-6, aPKC, and the exocyst are also required for proper intracellular lumen formation in Drosophila tracheal cells (Jones et al., 2014), suggesting that this pathway may function as a general mechanism promoting intracellular tube extension. Notably, and consistent with our findings in the $C$. elegans excretory cell, mutations in Drosophila baz (par-3) do not prevent tracheal lumenogenesis, suggesting that in both cell types PAR-6 and PKC-3/aPKC perform the major role in exocyst regulation. In addition, both PAR proteins and the exocyst are required for an organized lumen to form in cell cysts grown in 3D culture (Bryant et al., 2010). Thus PAR-mediated exocyst recruitment to sites of lumen formation, where additional membrane is needed, appears to be a feature common to both intracellular and multicellular tubes despite their dramatically different organization.

Exocyst recruitment by PAR proteins 
Together with previous studies, our findings suggest that PAR proteins and the exocyst may interface in multiple ways. In mammary epithelial cells, Par3 functions as an exocyst receptor, utilizing a lysine-rich domain to bind Exo70 and recruit the complex (Ahmed and Macara, 2017). However, in these cells, the exocyst also mediates membrane fusion at the basal membrane, where Par3 is not detected, suggesting that alternative exocyst receptors exist (Ahmed et al., 2018). Biochemical studies have also revealed interactions between the exocyst and PAR-6 and aPKC. For example, coimmunoprecipitation experiments in cultured rat kidney epithelial cells and in cortical neurons showed that aPKC immunoprecipitates with the exocyst proteins Sec8, Sec6, or Exo84 (Lalli, 2009; Rosse et al., 2009). Furthermore, Par6 can directly bind Exo84 in cultured mammalian neurons, and this interaction is promoted by the RAL-1 homologue RalA (Das et al., 2014). Finally, in rat kidney epithelial cells, aPKC helps recruit exocyst through the aPKC-interacting protein Kibra (Rosse et al., 2009). Together with these studies, our finding that PAR-6 but not PAR-3 is required to recruit SEC-10 to the lumenal membrane suggests that PAR-6 functions as an alternative means to recruit the exocyst complex to the membrane. Further studies will be needed to clarify whether it does so directly by functioning as an exocyst receptor, analogous to mammalian Par3 (Ahmed and Macara, 2017), or indirectly, for example through the kinase activity of aPKC. Because aPKC and Par6 localize interdependently in nearly all cell types examined, the fact that PKC-3 $3^{(\text {exc- })}$ and PAR-6 $6^{(\text {exc- })}$ larvae have similar lumen extension defects does not clarify how PKC-3 contributes to exocyst recruitment. Notably, C. elegans lacks a clear Kibra orthologue (Yoshihama et al., 2012), suggesting that if PKC3 interfaces with the exocyst directly, it does so utilizing a distinct mechanism. 


\section{MATERIALS AND METHODS}

C. elegans strains

Strains used in this study are listed in Table S1. All strains were cultured on Nematode Growth Medium (NGM) plates seeded with Escherichiae coli OP50 bacteria and maintained at $20^{\circ} \mathrm{C}$ unless specified otherwise (Brenner, 1974).

\section{Transgene construction}

All transgenes were constructed using Gibson assembly (Gibson et al., 2009) as follows:

pJA022 (t28h11.8p::mCherry) was assembled using vector pSA086 (pgp-12p::mCherry, Armenti et al. 2014b), and the $28 \mathrm{~h} 11.8 p$ promoter was amplified from genomic DNA.

$785 \mathrm{bp}$ of sequence upstream of the start codon of $t 28 \mathrm{~h} 11.8$ gene was used to generate the $t 28 h 11.8 p$ promoter.

pJA027 (t28h11.8p::zif-1) was assembled using vector pSA097 (pgp-12p::zif-1) containing zif-1 coding sequence, and the $t 28 h 11.8 p$ promoter sequence was added by Gibson assembly.

t28h11.8p::yfp and ifb-1::cfp were co-expressed in the same operon by inserting SL2 trans-splice acceptor sequences (244 bp intergenic sequence between gpd-2 stop codon and gpd-3 start site) between the yfp stop codon and the ifb-1 start codon (Tursun et al. 2009). pJA043 (t28h11.8p::yfp::s/2:::ifb-1::cfp) was assembled using vector pJA042 (t28h11.8p::ifb-1::cfp) which contains ifb-1 coding sequence; yfp and s/2 
were inserted between the promoter and ifb-1; s/2 was amplified from pJN645. yfp (pPD136.64) and cfp (pPD136.61) have synthetic introns (Fire lab vector kit).

pJA045 (hsp-16.41p::zif-1) was assembled using vector pSA120 which contains hsp16.41 promoter sequence (Armenti et al., 2014b; Hao et al., 2006), and zif-1 coding sequence was added by Gibson assembly.

pJA050 (t28h11.8p::cfp) was assembled using vector pJA027 (t28h11.8p::zif-1), and cfp was added by Gibson assembly.

\section{CRISPR knock-ins}

Plasmids for CRISPR/Cas9 genomic editing to make par-3(xn59[par-3::zf1::yfp loxP unc-119(+) loxP]) were constructed as described previously (Dickinson et al., 2013). The guide RNA sequence from plasmid pDD122 was replaced with the sequences (5'GTACTGGGGAAAACGATGAGG-3') and (5'-GAAGCCTACGAGACACGTGG-3') to create two single guide RNAs (sgRNAs) that cleave near the par-3 C-terminus (plasmids pJA029 and pJA030). A homologous repair plasmid for par-3 (pJA033) was constructed using Gibson assembly. The following DNA segments were assembled in order: 1179 bp upstream of par-3 stop codon (including ten silent point mutations adjacent to the predicted sgRNA cut sites) as the left homology arm; zf1::yfp with unc119; and the 3' terminal 932 bp of par-3 genomic sequence as the right homology arm. zf1::yfp with unc-119 flanked by LoxP sites was amplified from plasmid pJN601, which contains LoxP-flanked unc-119 inserted in reverse orientation into a synthetic intron 
within yfp (Armenti et al., 2014b). The vector backbone was PCR-amplified from pJN601 using Gibson assembly primers that overlapped with homology arms for par-3.

par-3(xn59: par-3-zf1-yfp + unc-119) was generated by microinjecting the sgRNA plasmids pJA029 and pJA030 (which also contains Cas9), the homologous repair template pJA033, and plasmid coinjection markers pGH8 (rab-3P::mCherry::unc-54utr; plasmid 19359; Addgene), pCFJ104 (myo-3P::mCherry::unc-54utr; plasmid 19328; Addgene), pCFJ90 (myo-2P::mCherry::unc-54utr; plasmid 19327; Addgene), and pMA122 (peel-1 negative selection; plasmid 34873; Addgene) into unc-119(ed3) mutant worms (Dickinson et al., 2013; Frokjaer-Jensen et al., 2012). Plates containing non-Unc $\mathrm{F} 2$ transformants were heat-shocked at $34^{\circ} \mathrm{C}$ for $4 \mathrm{~h}$ to activate PEEL-1 toxin in arraybearing animals, and successfully edited non-Unc animals were confirmed by the absence of mCherry expression in the F2 generation and YFP expression in their progeny.

exc-5(xn108[exc-5::zf1::mScarlet]) was generated by injecting a crRNA (IDT) with target homology sequence (5'-GAATCATCATTCAGATTGCT-3'). zf1::mScarlet dsDNA repair template with $\sim 120$ bp homology arms was prepared using primers (5'-

CGAATGTACACAATGACCGCTGAAGACGAACAAACCCAAATGAAATGGTTGGCGAT TTTGGATTTAGCCGCAAACGCACATCTGAAGAATCAACGGAATTCTGGATCCGAAC AGAGCGAACCGACAGAATACAAAACGCGAC-3'), which included five silent point mutations adjacent to the predicted crRNA cut sites, and (5'GAAAATTTGGATACAGTTTCAACGAACGAATAATAAGAATTGAGAGAAAAACAAGAA TAGAACACTGAAATAACTAAGAAAATAAACATATGTCTTGGCTGGGTGCCAAAAAA GAATCATCACTTGTAGAGCTCGTCCATTCCTC-3'), with plasmid pJA047 as a 
template. F1 worms with the co-CRISPR $d p y$-10(cn64) mutation (Paix et al., 2016) were screened by fluorescence and verified by PCR and sequencing.

Knock-in alleles were functional and viable, with only a minor level of lethality (par3(xn59), 97\% [353/363] viable; exc-5(xn108), 99\% [400/405] viable).

\section{Transgene integration}

pJA027 (t28h11.8p::zif-1), which contains an unc-119(+) transformation marker, was injected into unc-119(ed3) worms to obtain a stably inherited, high-copy extrachromosomal array. The array was integrated using Trioxsalen (Sigma) and UV irradiation. A mixed population of washed transgenic worms was incubated in $600 \mathrm{ml}$ of $33.3 \mathrm{ng} / \mathrm{ml}$ Trioxsalen in DMSO in the dark for $15 \mathrm{~min}$. Worms were dripped onto an unseeded NGM agar plate and, after the solution soaked in, the agar plate was irradiated with $360 \mu \mathrm{J}$ of UV light in a Stratalinker. NA22 bacterial food was dripped onto the worms and, after $5 \mathrm{~h}$ in darkness, 20 L4 stage transgenic worms were picked to each of 20 peptone plates $(10 \mathrm{~cm})$ seeded with NA22 bacteria. F1 adults were bleached to collect eggs, which were plated 200 per plate onto 70 NGM plates $(6 \mathrm{~cm})$. Nine hundred eighty-four transgenic F2s were picked into individual wells of 24-well plates, and those with an F3 brood containing only non-Unc progeny were saved. Transgenic insertion $x n / s 547$ was isolated and outcrossed three times to unc-119(ed3). 


\section{Imaging}

For all live-imaging experiments, larvae were mounted onto $5 \%$ agarose pads in a $2 \mathrm{mM}$ Levamisole solution in M9 buffer to induce paralysis. Fluorescent images were acquired using an SP8 confocal microscope (Leica), 63x 1.4 NA oil-immersion objective, 458, 488-, 514-, $561 \mathrm{~nm}$ lasers, and 1-5x zoom. For intensity measurements, larvae were imaged using HyD detectors and the photon-counting mode. Images were analyzed and processed in ImageJ $(\mathrm{NIH})$ with no $\gamma$ adjustments and level adjustments across pixels. For quantifications, the same laser power and exposure times were used within experiments and control and mutant images were processed similarly. After processing in ImageJ, images were rotated and cropped using Illustrator (CC2020, Adobe).

Fluorescence images for Figure 1D, Figure S1, and Figure S3 were acquired on an Axio Imager.A2 microscope (Zeiss) with $63 \times 1.4$ NA or $40 \times 1.3$ NA objective and a CCD camera (model C10600-10B-H, S. 160522; Hamamatsu). Images were processed using the unsharpen mask method in ImageJ.

Heat-shock expression of ZIF-1

Plates containing late L4/young adult animals were placed in a water bath at $34^{\circ} \mathrm{C}$ for 30 minutes and then transferred to $15^{\circ} \mathrm{C}$ to recover. In each experiment, control and experimental animals were imaged 2-4 hours following heat shock. 


\section{Excretory canal outgrowth measurements}

Excretory canal length was scored visually using a canal-specific cytoplasmic marker (t28h11.8p::yfp) at L1 and L4 larval stages. Both posterior canal arms were scored in each animal. In cases where the canal arms differed in length, an approximate average of the two lengths was recorded for that animal.

Genetic test of ZIF-1 degradation

To generate SEC-5 exc(-)/sec-5(tm1443): sec-5(tm1443)/mIn1 males were mated with sec-5(xn51); xnls547[t28h11.8p::zif-1] hermaphrodites that contain the $x n E x 466$ extrachromosomal array marking the canal lumen and cytoplasm. Canal length in Figure S2 was scored in F1 generation male cross progeny that did not carry the $m \ln 1$ balancer [genotype was sec-5(xn51)/sec-5(tm1443); xnls547[t28h11.8p::zif-1]/+].

Controls were generated by mating sec-5(xn51) males with sec-5(xn51); $x n / s 547[t 28 h 11.8 p:: z i f-1]$ hermaphrodites that carried the xnEx466 extrachromosomal array. Canal length of controls was scored in F1 generation male cross progeny [genotype was sec-5(xn51); xnls547[t28h11.8p::zif-1]/+].

Image analysis

All measurements were performed using ImageJ and raw SP8 confocal image files. 
For lumen/cytoplasm intensity measurements, a line four pixels in width was drawn along the lumenal membrane and a second line was drawn along an adjacent region within the canal cytoplasm, as shown in Figure 5A. Mean pixel intensity values along each line were calculated using the ImageJ measuring tool. Both faces of the lumenal membrane were measured in each image and two images were acquired of different regions of the posterior canal arms within each animal. Four such measurements were taken for each animal and an average 'lumen/cytoplasm intensity ratio' was calculated, which is represented by small colored dots in plots in Figure 5F, 5K, 6E, 6J, 7E, 7J.

For intensity profiles of the excretory canal, a line 30 pixels in width was drawn across a $3 \mu \mathrm{m}$ region of the excretory canal cytoplasm, as shown in Figure 3D. Three measurements were acquired for each animal and averaged to generate a single intensity profile per animal. Measurements from five animals are shown in each graph. Values were copied into GraphPad Prism 8 to generate an XY line plot displaying the average and standard deviation.

To measure excretory canal fluorescence intensity after ZIF-1 degradation, the polygon tool in ImageJ was used to draw a region of interest (ROI) around the canal cytoplasm using the CFPcytoplasm marker. Mean pixel intensity values within each polygon were calculated using the ImageJ measuring tool. To measure degradation, fluorescent intensity of PAR-6::ZF1::YFP was calculated in control and $h s p P:: z i f-1$ animals 2 hours after a 30 minute heat shock at $34^{\circ} \mathrm{C}$. Two images were acquired of different regions of the posterior canal arms of each animal and averaged. Background YFP autofluorescence was calculated in wild type larvae carrying the pgp-12p::mCherry transgene to mark canal cytoplasm. Average background autofluorescence was 
subtracted from control and $h s p P:: z i f-1$ animals prior to calculating percent of YFP depletion. Error bars represent standard deviation, and were calculated from the change in mean fluorescence intensity between control and experimental animals.

For plotting image quantification and statistical analysis, mean values for each animal and each biological replicate were copied to GraphPad Prism 8. SuperPlots were generated in GraphPad Prism 8 as previously described (Lord et al., 2020), with dots of the same color representing individual data points from the same experiment.

\section{Statistics}

Statistical analysis was performed in GraphPad Prism 8. Statistical tests, number of embryos, and number of experiments are indicated in the figure legends. In Figure 2, where multiple comparisons were made to a common control, $p$-values were corrected using the Bonferroni method.

\section{ACKNOWLEDGEMENTS}

We thank Ken Kemphues, Dan Dickinson, and Bob Goldstein for generous gifts of worm strains, Steve Armenti for plasmids used in transgene construction, and members of the Nance laboratory and Jane Hubbard for comments. Some strains were provided by the CGC, which is funded by NIH Office of Research Infrastructure Programs (P40 OD010440). This work was supported by fellowships from the American Cancer Society and the National Institutes of Health to J.A. (PF-16-175-01-DDC and F32HL136038) and research grants from the National Institutes of Health to J.N. (R01GM098492 and R35GM118081). 


\section{FIGURE LEGENDS}

\section{Figure 1. SEC-5 and RAL-1 are required in the excretory cell for lumen extension}

(A) Schematics of L4 larval stage worms depicting excretory cell-specific protein depletion using excP::zif-1. The $\mathrm{H}$-shaped excretory canal is outlined and a hypothetical ubiquitous ZF1-tagged protein is depicted in green. The typical region of the canal examined by microscopy is enlarged to show cytoplasmic (yellow, excP::YFP) and lumenal (cyan, IFB-1::CFP) markers used for analyzing excretory canal morphology. Anterior left, dorsal top. (B and C) L4 stage excretory canal in transgenic control (B) and excP::zif-1 (C) animals expressing ZF1::GFP::CDC-42 (C). Outline of excretory canal cytoplasm is indicated by dotted line. ZF1::GFP::CDC-42 is degraded in the excretory cell, but not surrounding cells (arrowhead), in excP::zif-1 animals. (D) Endogenous expression of SEC-5::ZF1::YFP at the excretory canal lumen of L4 stage larva. (E-J") Larval excretory canal phenotypes in control (E-F"), SEC-5 ${ }^{\text {exc(-) }}\left(\mathrm{G}-\mathrm{H}^{\prime \prime}\right)$, and RAL-1 ${ }^{\mathrm{exc}(-)}$ (I-J'). Canal cytoplasm and lumen is marked by an extrachromosomal array expressing excretory cell-specific cytoplasmic and lumenal markers (see panel A). Confocal images were acquired using 20x (E, G, I) and 63x objectives (F-F", H-H", J-J'). Excretory cell body indicated by asterisk. Posterior tip of excretory canal indicated by white arrow. Posterior excretory canal that has extended beyond the focal plane is indicated by dashed white arrow. Dashed box indicates approximate region represented in high magnification images. Outline of each animal is indicated by solid white line. Scale bars, $10 \mu \mathrm{m}$. 
Figure 2. Canal outgrowth phenotypes upon exocyst or PAR protein depletion

Schematics of the excretory cell are shown at the L1 stage, when the canal is extending, and the L4 larval stage, when the canal is fully extended. Canal outgrowth defects upon depleting the indicated proteins in the excretory cell are depicted as the percentage of animals in each of four phenotypic categories that measure posterior canal extension relative to body length. The relative intensity of green shading reflects the percentage of larvae observed in each phenotypic category. $P$-values were calculated using Fisher's exact test ( $\mathrm{L} 1$ stage: $<50 \%$ versus $>50 \%$ canal outgrowth; $L 4$ stage: $<75 \%$ versus $>75 \%$ canal outgrowth). $P$-value significance was adjusted using Bonferroni correction to account for multiple comparisons to a common control, such that $P \leq 0.008$ is considered statistically significant.

Figure 3. PAR-6, PKC-3, and PAR-3 are enriched at the lumenal membrane and CDC-42 extends into the canal cytoplasm

(A-C) Distribution of endogenously tagged PAR-6, PKC-3 and PAR-3 in the excretory cell canal. (D) Schematic of excretory cell line trace measurements displayed in $(F)$ and $(H)$. Three line-trace measurements $\left(m_{1}, m_{2}, m_{3}\right)$ were taken perpendicular to the excretory cell lumen in each animal. Measurements were averaged to generate a single line trace for each larva, and five larvae were measured from each genotype. (E-E') Distribution of PAR-6::ZF1::YFP and PAR-3::mCherry in the larval excretory canal. (F) Line traces of PAR-6::ZF1::YFP (green) and PAR-3::mCherry (magenta). Solid line represents mean and shaded area is $\pm S D$. Intensities were normalized to compare peak values of each channel. '0.0' on $\mathrm{x}$-axis represents the center point of the canal lumen. $n$ 
= 5 larvae. (G-G”) Distribution of ZF1::YFP::CDC-42 and PAR-6::mKate in the larval excretory canal. (H) Line trace of ZF1::YFP::CDC-42 (green) and PAR-6::mKate (magenta). Solid line represents mean and shaded area is $\pm S D$. Intensities were normalized to compare peak values of each channel. '0.0' on $\mathrm{x}$-axis represents the center point of the canal lumen. $n=5$ larvae. Outline of excretory canal cytoplasm is indicated by dashed lines. Scale bars, $10 \mu \mathrm{m}$.

Figure 4. PAR-6, PKC-3 and CDC-42, but not PAR-3, are required for excretory cell lumen extension

Larval excretory canal phenotypes in PAR-6 ${ }^{\operatorname{exc}(-)}\left(A-B^{\prime \prime}\right)$, PKC-3 ${ }^{\operatorname{exc}(-)}\left(C-D^{\prime \prime}\right), C D C-42^{\operatorname{exc}(-)}$ (E-F') and PAR-3exc(-) (G-H') L4 stage worms expressing cytoplasmic and lumenal markers. Confocal images were acquired using 20x (A, C, E, G) and 63x (B-B"', D-D”, F-F”, H-H”) objectives. Excretory cell body indicated by asterisk. Posterior tip of excretory canal indicated by white arrow. Posterior excretory canal that has extended beyond the focal plane is indicated by dashed white arrow. Dashed box indicates region represented in high magnification images. Outline of each animal is indicated by solid white line. Scale bars, $10 \mu \mathrm{m}$.

Figure 5. PAR-6, but not PAR-3, is required to enrich SEC-10 at the lumenal membrane

(A) Schematic of L4 larval stage worms depicting heat-shock inducible protein depletion. The excretory canal is outlined in black and a hypothetical ubiquitous ZF1tagged protein is shown in green. Upon heat-shock, the ZF1-tagged protein is rapidly 
degraded in all somatic cells of animals expressing $h s p P:: z i f-1$. To measure fluorescence intensity, average pixel intensity was calculated along a region of the excretory cell lumen ('L') and within the cytoplasm ('C'); dividing L/C yields the lumen/cytoplasm ratio shown in $(F)$ and $(K)$. Anterior left, dorsal top. (B-C) Distribution of PAR-6::ZF1::YFP in larval excretory canal in control (B) and hspP::zif-1 (C). (B'-C') Distribution of mCherry::SEC-10 in larval excretory canal in PAR-6::ZF1::YFP control (B') and $h s p P:: z i f-1$ (C'). (D-E) Line trace of PAR-6::ZF1::YFP (green) and mCherry::SEC-10 (magenta). Intensities were normalized to compare peak values of each channel. '0.0' on $\mathrm{x}$-axis represents the center point of the canal lumen. $n=5$ larvae. (F) Quantification of lumenal membrane to cytoplasm intensity ratio of mCherry::SEC-10 in the excretory canal of control and hspP::zif-1 larvae expressing PAR-6::ZF1::YFP. Individual data points (small dots) are color-coded (orange, purple, and light blue) from three independent replicates. Large dots represent the mean of each replicate, horizontal bar is the mean of means, and error bars are the SEM. $P$ values were calculated using a ratio paired t-test of the means. $n=5,8,7$ for control; $n$ =13, 11, 10 for hspP::zif-1. (G-H) Distribution of PAR-3::ZF1::YFP in larval excretory canal in control $(\mathrm{G})$ and $h \operatorname{sp} P:: z i f-1(\mathrm{H}) .\left(\mathrm{G}^{\prime}-\mathrm{H}^{\prime}\right)$ Distribution of mCherry::SEC-10 in the larval excretory canal of control (G') and hspP::zif-1 worms expressing PAR3::ZF1::YFP (H'). (I-J) Line trace of PAR-3::ZF1::YFP (green) and mCherry::SEC-10 (magenta). Intensities were normalized to compare peak values of each channel. ' 0.0 ' on x-axis represents the center point of the canal lumen. $n=5$ larvae. (K) Quantification of lumenal membrane to cytoplasm intensity ratio of mCherry::SEC-10 expression in the excretory canal of control and $h s p P:: z i f-1$ larvae expressing PAR-3::ZF1::YFP. Data is 
shown as in panel F. P-values were calculated using a ratio paired t-test of the means. $n=7,9,8$ for control; $n=7,8,8$ for $h s p P:: z i f-1$. Outline of excretory canal cytoplasm is indicated by dashed line. Scale bars, $10 \mu \mathrm{m}$.

\section{Figure 6. PAR-3 is required to enrich PAR-6 at the lumenal membrane}

(A-B) Distribution of PAR-3::ZF1::YFP in larval excretory canal in control (A) and hspP::zif-1 (B) worms. (A'-B') Distribution of PAR-6::mKate in the larval excretory canal of control (A') and hspP::zif-1 (B') worms expressing PAR-3::ZF1::YFP. Arrowheads show punctate PAR-6::mKate along lumenal membrane. (C-D) Line traces of PAR3::ZF1::YFP (green) and PAR-6::mKate (magenta). Intensities were normalized to compare peak values of each channel. '0.0' on $x$-axis represents the center point of the canal lumen. $n=5$ larvae. (E) Quantification of lumenal membrane to cytoplasm intensity ratio of PAR-6::mKate expression in the excretory canal of control and hspP::zif-1 larvae expressing PAR-3::ZF1::YFP. Individual data points (small dots) are color-coded (orange, purple, and light blue) from three independent replicates. Large dots represent the mean of each replicate, horizontal bar is the mean of means, and error bars are the SEM. P-values were calculated using a ratio paired t-test of the means. $n=6,6$, 8 for control; $n=4,7,8$ for $h s p P:: z i f-1$. (F-G) Distribution of PAR6::ZF1::YFP in larval excretory canal in control (F) and hspP::zif-1 (G) worms. (F'-G') Distribution of PAR-3::mCherry in larval excretory canal of control (F') and hspP::zif-1 (G') worms expressing PAR-6::ZF1::YFP. (H-I) Line traces of PAR-6::ZF1::YFP (green) and PAR-3::mCherry (magenta). Intensities were normalized to compare peak values of each channel. '0.0' on $\mathrm{x}$-axis represents the center point of the canal lumen. $n=5$ for 
control; $n=5$ for $h s p P:: z i f-1$. (J) Quantification of lumenal membrane to cytoplasm intensity ratio of PAR-3::mCherry expression in the excretory canal of control and hspP::zif-1 larvae expressing PAR-6::ZF1::YFP. Data depicted as in panel E. P-values were calculated using a ratio paired t-test of the means. $n=9,8,9$ for control; $n=7,8$, 9 for $h s p P:: z i f-1$. Outline of excretory canal cytoplasm is indicated by dotted line. Scale bars, $10 \mu \mathrm{m}$.

Figure 7. CDC-42 and EXC-5 are required to enrich PAR-6 and PKC-3 at the lumenal membrane

(A-B) Distribution of ZF1::YFP::CDC-42 in larval excretory canal in control (A) and hspP::zif-1 (B) worms. (A'-B') Distribution of PAR-6::mKate in the larval excretory canal of control (A') and hspP::zif-1 (B') worms expressing ZF1::YFP::CDC-42. (C-D) Line trace of ZF1::YFP::CDC-42 (green) and PAR-6::mKate (magenta). Intensities were normalized to compare peak values of each channel. '0.0' on $x$-axis represents the center point of the canal lumen. $n=5$ larvae. (E) Quantification of lumenal membrane to cytoplasm intensity ratio of PAR-6::mKate expression in the excretory canal of control and $h s p P:: z i f-1$ larvae expressing ZF1::YFP::CDC-42. Individual data points (small dots) are color-coded (orange, purple, and light blue) from three independent replicates. Large dots represent the mean of each replicate, horizontal bar is the mean of means, and error bars are the SEM. $P$-values were calculated using a ratio paired t-test of the means. $n=8,7,7$ for control; $n=9,7,8$ for $h s p P:: z i f-1$. (F-G) Distribution EXC5::ZF1::mScarlet in the larval excretory canal in control (F) and $h s p P:: z i f-1(\mathrm{G})$ worms. (F'-G') Distribution of GFP::PKC-3 in the larval excretory canal of control (F') and 
hspP::zif-1 (G') worms expressing EXC-5::ZF1::mScarlet. (H-I) Line trace of GFP::PKC3 (green) and EXC-5::ZF1::mScarlet (magenta). Intensities were normalized to compare peak values of each channel. '0.0' on x-axis represents the center point of the canal lumen. $n=5$ larvae. (J) Quantification of lumenal membrane to cytoplasm intensity ratio of GFP::PKC-3 expression in the excretory canal of control and hspP::zif-1 larvae expressing ZF1::YFP::CDC-42. Data are depicted as in panel E. $P$-values were calculated using a ratio paired t-test of the means. $n=5,6,6$ for control; $n=5,5,6$ for hspP::zif-1. (K) Model of PAR and exocyst regulation of excretory cell lumen extension. Cross section of larval excretory canal (left) depicts large, canalicular vesicles fusing with the lumenal membrane (red) during lumen extension. Boxed region represents a portion of canal where lumen extension is occurring, magnified at right to show a proposed molecular pathway for lumenal vesicle tethering. Outline of excretory canal cytoplasm is indicated by dotted line. Scale bars, $10 \mu \mathrm{m}$. 


\section{REFERENCES}

Aceto, D., M. Beers, and K.J. Kemphues. 2006. Interaction of PAR-6 with CDC-42 is required for maintenance but not establishment of PAR asymmetry in C. elegans. Developmental biology. 299:386-397.

Achilleos, A., A.M. Wehman, and J. Nance. 2010. PAR-3 mediates the initial clustering and apical localization of junction and polarity proteins during C. elegans intestinal epithelial cell polarization. Development. 137:1833-1842.

Ahmed, S.M., and I.G. Macara. 2017. The Par3 polarity protein is an exocyst receptor essential for mammary cell survival. Nature communications. 8:14867.

Ahmed, S.M., H. Nishida-Fukuda, Y. Li, W.H. McDonald, C.C. Gradinaru, and I.G. Macara. 2018. Exocyst dynamics during vesicle tethering and fusion. Nature communications. 9:5140.

Armenti, S.T., E. Chan, and J. Nance. 2014a. Polarized exocyst-mediated vesicle fusion directs intracellular lumenogenesis within the C. elegans excretory cell. Developmental biology. 394:110-121.

Armenti, S.T., L.L. Lohmer, D.R. Sherwood, and J. Nance. 2014b. Repurposing an endogenous degradation system for rapid and targeted depletion of C. elegans proteins. Development. 141:4640-4647.

Beers, M., and K. Kemphues. 2006. Depletion of the co-chaperone CDC-37 reveals two modes of PAR-6 cortical association in C. elegans embryos. Development. 133:3745-3754.

Berry, K.L., H.E. Bulow, D.H. Hall, and O. Hobert. 2003. A C. elegans CLIC-like protein required for intracellular tube formation and maintenance. Science. 302:21342137. 
Brenner, S. 1974. The genetics of Caenorhabditis elegans. Genetics. 77:71-94.

Bryant, D.M., A. Datta, A.E. Rodriguez-Fraticelli, J. Peranen, F. Martin-Belmonte, and K.E. Mostov. 2010. A molecular network for de novo generation of the apical surface and lumen. Nature cell biology. 12:1035-1045.

Brymora, A., V.A. Valova, M.R. Larsen, B.D. Roufogalis, and P.J. Robinson. 2001. The brain exocyst complex interacts with RalA in a GTP-dependent manner: identification of a novel mammalian Sec3 gene and a second Sec15 gene. The Journal of biological chemistry. 276:29792-29797.

Buechner, M., D.H. Hall, H. Bhatt, and E.M. Hedgecock. 1999. Cystic canal mutants in Caenorhabditis elegans are defective in the apical membrane domain of the renal (excretory) cell. Developmental biology. 214:227-241.

Chen, X.W., D. Leto, J. Xiao, J. Goss, Q. Wang, J.A. Shavit, T. Xiong, G. Yu, D. Ginsburg, D. Toomre, Z. Xu, and A.R. Saltiel. 2011. Exocyst function is regulated by effector phosphorylation. Nature cell biology. 13:580-588.

Das, A., S. Gajendra, K. Falenta, M.J. Oudin, P. Peschard, S. Feng, B. Wu, C.J. Marshall, P. Doherty, W. Guo, and G. Lalli. 2014. RalA promotes a direct exocyst-Par6 interaction to regulate polarity in neuronal development. Journal of cell science. 127:686-699.

Denker, E., I. Bocina, and D. Jiang. 2013. Tubulogenesis in a simple cell cord requires the formation of bi-apical cells through two discrete Par domains. Development. 140:2985-2996.

DeRenzo, C., K.J. Reese, and G. Seydoux. 2003. Exclusion of germ plasm proteins from somatic lineages by cullin-dependent degradation. Nature. 424:685-689. 
Dickinson, D.J., F. Schwager, L. Pintard, M. Gotta, and B. Goldstein. 2017. A SingleCell Biochemistry Approach Reveals PAR Complex Dynamics during Cell Polarization. Developmental cell. 42:416-434 e411.

Dickinson, D.J., J.D. Ward, D.J. Reiner, and B. Goldstein. 2013. Engineering the Caenorhabditis elegans genome using Cas9-triggered homologous recombination. Nature methods. 10:1028-1034.

Frische, E.W., W. Pellis-van Berkel, G. van Haaften, E. Cuppen, R.H. Plasterk, M. Tijsterman, J.L. Bos, and F.J. Zwartkruis. 2007. RAP-1 and the RAL-1/exocyst pathway coordinate hypodermal cell organization in Caenorhabditis elegans. The EMBO journal. 26:5083-5092.

Frokjaer-Jensen, C., M.W. Davis, M. Ailion, and E.M. Jorgensen. 2012. Improved Mos1mediated transgenesis in C. elegans. Nature methods. 9:117-118.

Gao, J., L. Estrada, S. Cho, R.E. Ellis, and J.L. Gorski. 2001. The Caenorhabditis elegans homolog of FGD1, the human Cdc42 GEF gene responsible for faciogenital dysplasia, is critical for excretory cell morphogenesis. Human molecular genetics. 10:3049-3062.

Gervais, L., and J. Casanova. 2010. In vivo coupling of cell elongation and lumen formation in a single cell. Current biology : CB. 20:359-366.

Gibson, D.G., L. Young, R.Y. Chuang, J.C. Venter, C.A. Hutchison, 3rd, and H.O. Smith. 2009. Enzymatic assembly of DNA molecules up to several hundred kilobases. Nature methods. 6:343-345.

Gotta, M., M.C. Abraham, and J. Ahringer. 2001. CDC-42 controls early cell polarity and spindle orientation in C. elegans. Current biology : CB. 11:482-488. 
Hao, Y., L. Boyd, and G. Seydoux. 2006. Stabilization of cell polarity by the C. elegans RING protein PAR-2. Developmental cell. 10:199-208.

Hayakawa, M., M. Matsushima, H. Hagiwara, T. Oshima, T. Fujino, K. Ando, K. Kikugawa, H. Tanaka, K. Miyazawa, and M. Kitagawa. 2008. Novel insights into FGD3, a putative GEF for Cdc42, that undergoes SCF(FWD1/beta-TrCP)mediated proteasomal degradation analogous to that of its homologue FGD1 but regulates cell morphology and motility differently from FGD1. Genes Cells. 13:329-342.

He, B., and W. Guo. 2009. The exocyst complex in polarized exocytosis. Current opinion in cell biology. 21:537-542.

Heider, M.R., M. Gu, C.M. Duffy, A.M. Mirza, L.L. Marcotte, A.C. Walls, N. Farrall, Z. Hakhverdyan, M.C. Field, M.P. Rout, A. Frost, and M. Munson. 2016. Subunit connectivity, assembly determinants and architecture of the yeast exocyst complex. Nat Struct Mol Biol. 23:59-66.

Herwig, L., Y. Blum, A. Krudewig, E. Ellertsdottir, A. Lenard, H.G. Belting, and M. Affolter. 2011. Distinct cellular mechanisms of blood vessel fusion in the zebrafish embryo. Current biology : CB. 21:1942-1948.

Huber, C., A. Martensson, G.M. Bokoch, D. Nemazee, and A.L. Gavin. 2008. FGD2, a CDC42-specific exchange factor expressed by antigen-presenting cells, localizes to early endosomes and active membrane ruffles. The Journal of biological chemistry. 283:34002-34012. 
Hutterer, A., J. Betschinger, M. Petronczki, and J.A. Knoblich. 2004. Sequential roles of Cdc42, Par-6, aPKC, and Lgl in the establishment of epithelial polarity during Drosophila embryogenesis. Developmental cell. 6:845-854.

Joberty, G., C. Petersen, L. Gao, and I.G. Macara. 2000. The cell-polarity protein Par6 links Par3 and atypical protein kinase C to Cdc42. Nature cell biology. 2:531-539. Johansson, A., M. Driessens, and P. Aspenstrom. 2000. The mammalian homologue of the Caenorhabditis elegans polarity protein PAR-6 is a binding partner for the Rho GTPases Cdc42 and Rac1. Journal of cell science. 113 ( Pt 18):3267-3275. Jones, T.A., and M.M. Metzstein. 2011. A novel function for the PAR complex in subcellular morphogenesis of tracheal terminal cells in Drosophila melanogaster. Genetics. 189:153-164.

Jones, T.A., L.S. Nikolova, A. Schjelderup, and M.M. Metzstein. 2014. Exocystmediated membrane trafficking is required for branch outgrowth in Drosophila tracheal terminal cells. Developmental biology. 390:41-50.

Kay, A.J., and C.P. Hunter. 2001. CDC-42 regulates PAR protein localization and function to control cellular and embryonic polarity in C. elegans. Current biology : CB. 11:474-481.

Kemphues, K.J., J.R. Priess, D.G. Morton, and N.S. Cheng. 1988. Identification of genes required for cytoplasmic localization in early C. elegans embryos. Cell. $52: 311-320$

Khan, L.A., H. Zhang, N. Abraham, L. Sun, J.T. Fleming, M. Buechner, D.H. Hall, and V. Gobel. 2013. Intracellular lumen extension requires ERM-1-dependent apical membrane expansion and AQP-8-mediated flux. Nature cell biology. 15:143-156. 
Kolotuev, I., V. Hyenne, Y. Schwab, D. Rodriguez, and M. Labouesse. 2013. A pathway for unicellular tube extension depending on the lymphatic vessel determinant Prox1 and on osmoregulation. Nature cell biology. 15:157-168.

Kurogane, Y., M. Miyata, Y. Kubo, Y. Nagamatsu, R.K. Kundu, A. Uemura, T. Ishida, T. Quertermous, K. Hirata, and Y. Rikitake. 2012. FGD5 mediates proangiogenic action of vascular endothelial growth factor in human vascular endothelial cells. Arterioscler Thromb Vasc Biol. 32:988-996.

Lalli, G. 2009. RalA and the exocyst complex influence neuronal polarity through PAR-3 and aPKC. Journal of cell science. 122:1499-1506.

Lant, B., B. Yu, M. Goudreault, D. Holmyard, J.D. Knight, P. Xu, L. Zhao, K. Chin, E. Wallace, M. Zhen, A.C. Gingras, and W.B. Derry. 2015. CCM-3/STRIPAK promotes seamless tube extension through endocytic recycling. Nature communications. 6:6449.

Lenard, A., E. Ellertsdottir, L. Herwig, A. Krudewig, L. Sauteur, H.G. Belting, and M. Affolter. 2013. In vivo analysis reveals a highly stereotypic morphogenetic pathway of vascular anastomosis. Developmental cell. 25:492-506.

Lin, D., A.S. Edwards, J.P. Fawcett, G. Mbamalu, J.D. Scott, and T. Pawson. 2000. A mammalian PAR-3-PAR-6 complex implicated in Cdc42/Rac1 and aPKC signalling and cell polarity. Nature cell biology. 2:540-547.

Lipschutz, J.H., W. Guo, L.E. O'Brien, Y.H. Nguyen, P. Novick, and K.E. Mostov. 2000. Exocyst is involved in cystogenesis and tubulogenesis and acts by modulating synthesis and delivery of basolateral plasma membrane and secretory proteins. Molecular biology of the cell. 11:4259-4275. 
Liu, J., and W. Guo. 2012. The exocyst complex in exocytosis and cell migration. Protoplasma. 249:587-597.

Lord, S.J., K.B. Velle, R.D. Mullins, and L.K. Fritz-Laylin. 2020. SuperPlots: Communicating reproducibility and variability in cell biology. The Journal of cell biology. 219.

Lubarsky, B., and M.A. Krasnow. 2003. Tube morphogenesis: making and shaping biological tubes. Cell. 112:19-28.

Mancuso, V.P., J.M. Parry, L. Storer, C. Poggioli, K.C. Nguyen, D.H. Hall, and M.V. Sundaram. 2012. Extracellular leucine-rich repeat proteins are required to organize the apical extracellular matrix and maintain epithelial junction integrity in C. elegans. Development. 139:979-990.

Mano, I., S. Straud, and M. Driscoll. 2007. Caenorhabditis elegans glutamate transporters influence synaptic function and behavior at sites distant from the synapse. The Journal of biological chemistry. 282:34412-34419.

Mattingly, B.C., and M. Buechner. 2011. The FGD homologue EXC-5 regulates apical trafficking in C. elegans tubules. Developmental biology. 359:59-72.

Miyamoto, Y., J. Yamauchi, and H. Itoh. 2003. Src kinase regulates the activation of a novel FGD-1-related Cdc42 guanine nucleotide exchange factor in the signaling pathway from the endothelin A receptor to JNK. The Journal of biological chemistry. 278:29890-29900.

Montoyo-Rosario, J.G., S.T. Armenti, Y. Zilberman, and J. Nance. 2020. The Role of pkc-3 and Genetic Suppressors in Caenorhabditis elegans Epithelial Cell Junction Formation. Genetics. 214:941-959. 
Moskalenko, S., D.O. Henry, C. Rosse, G. Mirey, J.H. Camonis, and M.A. White. 2002. The exocyst is a Ral effector complex. Nature cell biology. 4:66-72.

Moskalenko, S., C. Tong, C. Rosse, G. Mirey, E. Formstecher, L. Daviet, J. Camonis, and M.A. White. 2003. Ral GTPases regulate exocyst assembly through dual subunit interactions. The Journal of biological chemistry. 278:51743-51748.

Nance, J., and C. Frokjaer-Jensen. 2019. The Caenorhabditis elegans Transgenic Toolbox. Genetics. 212:959-990.

Nance, J., and J.A. Zallen. 2011. Elaborating polarity: PAR proteins and the cytoskeleton. Development. 138:799-809.

Nelson, F.K., P.S. Albert, and D.L. Riddle. 1983. Fine structure of the Caenorhabditis elegans secretory-excretory system. J Ultrastruct Res. 82:156-171.

Nelson, F.K., and D.L. Riddle. 1984. Functional study of the Caenorhabditis elegans secretory-excretory system using laser microsurgery. The Journal of experimental zoology. 231:45-56.

Paix, A., H. Schmidt, and G. Seydoux. 2016. Cas9-assisted recombineering in C. elegans: genome editing using in vivo assembly of linear DNAs. Nucleic Acids Res. 44:e128.

Qiu, R.G., A. Abo, and G. Steven Martin. 2000. A human homolog of the C. elegans polarity determinant Par-6 links Rac and Cdc42 to PKCzeta signaling and cell transformation. Current biology : CB. 10:697-707.

Rasmussen, J.P., K. English, J.R. Tenlen, and J.R. Priess. 2008. Notch signaling and morphogenesis of single-cell tubes in the $C$. elegans digestive tract. Developmental cell. 14:559-569. 
Reese, K.J., M.A. Dunn, J.A. Waddle, and G. Seydoux. 2000. Asymmetric segregation of PIE-1 in C. elegans is mediated by two complementary mechanisms that act through separate PIE-1 protein domains. Molecular cell. 6:445-455.

Rodriguez, J., F. Peglion, J. Martin, L. Hubatsch, J. Reich, N. Hirani, A.G. Gubieda, J. Roffey, A.R. Fernandes, D. St Johnston, J. Ahringer, and N.W. Goehring. 2017. aPKC Cycles between Functionally Distinct PAR Protein Assemblies to Drive Cell Polarity. Developmental cell. 42:400-415 e409.

Rosse, C., E. Formstecher, K. Boeckeler, Y. Zhao, J. Kremerskothen, M.D. White, J.H. Camonis, and P.J. Parker. 2009. An aPKC-exocyst complex controls paxillin phosphorylation and migration through localised JNK1 activation. PLoS biology. 7:e1000235.

Schottenfeld-Roames, J., and A.S. Ghabrial. 2012. Whacked and Rab35 polarize dynein-motor-complex-dependent seamless tube growth. Nature cell biology. 14:386-393.

Shaye, D.D., and I. Greenwald. 2016. A network of conserved formins, regulated by the guanine exchange factor EXC-5 and the GTPase CDC-42, modulates tubulogenesis in vivo. Development. 143:4173-4181.

St Johnston, D., and J. Ahringer. 2010. Cell polarity in eggs and epithelia: parallels and diversity. Cell. 141:757-774.

Steenblock, C., T. Heckel, C. Czupalla, A.I. Espirito Santo, C. Niehage, M. Sztacho, and B. Hoflack. 2014. The Cdc42 guanine nucleotide exchange factor FGD6 coordinates cell polarity and endosomal membrane recycling in osteoclasts. The Journal of biological chemistry. 289:18347-18359. 
Stone, C.E., D.H. Hall, and M.V. Sundaram. 2009. Lipocalin signaling controls unicellular tube development in the Caenorhabditis elegans excretory system. Developmental biology. 329:201-211.

Sugihara, K., S. Asano, K. Tanaka, A. Iwamatsu, K. Okawa, and Y. Ohta. 2002. The exocyst complex binds the small GTPase RalA to mediate filopodia formation. Nature cell biology. 4:73-78.

Sundaram, M.V., and M. Buechner. 2016. The Caenorhabditis elegans Excretory System: A Model for Tubulogenesis, Cell Fate Specification, and Plasticity. Genetics. 203:35-63.

Sundaram, M.V., and J.D. Cohen. 2017. Time to make the doughnuts: Building and shaping seamless tubes. Semin Cell Dev Biol. 67:123-131.

Suzuki, N., M. Buechner, K. Nishiwaki, D.H. Hall, H. Nakanishi, Y. Takai, N. Hisamoto, and K. Matsumoto. 2001. A putative GDP-GTP exchange factor is required for development of the excretory cell in Caenorhabditis elegans. EMBO reports. 2:530-535.

Tabuse, Y., Y. Izumi, F. Piano, K.J. Kemphues, J. Miwa, and S. Ohno. 1998. Atypical protein kinase $\mathrm{C}$ cooperates with PAR-3 to establish embryonic polarity in Caenorhabditis elegans. Development. 125:3607-3614.

Umikawa, M., H. Obaishi, H. Nakanishi, K. Satoh-Horikawa, K. Takahashi, I. Hotta, Y. Matsuura, and Y. Takai. 1999. Association of frabin with the actin cytoskeleton is essential for microspike formation through activation of Cdc42 small G protein. The Journal of biological chemistry. 274:25197-25200. 
Wang, S.C., T.Y.F. Low, Y. Nishimura, L. Gole, W. Yu, and F. Motegi. 2017. Cortical forces and CDC-42 control clustering of PAR proteins for Caenorhabditis elegans embryonic polarization. Nature cell biology. 19:988-995.

Watts, J.L., B. Etemad-Moghadam, S. Guo, L. Boyd, B.W. Draper, C.C. Mello, J.R. Priess, and K.J. Kemphues. 1996. par-6, a gene involved in the establishment of asymmetry in early $C$. elegans embryos, mediates the asymmetric localization of PAR-3. Development. 122:3133-3140.

Wu, B., and W. Guo. 2015. The Exocyst at a Glance. Journal of cell science. 128:29572964.

Yoshihama, Y., K. Chida, and S. Ohno. 2012. The KIBRA-aPKC connection: A potential regulator of membrane trafficking and cell polarity. Commun Integr Biol. 5:146151.

Zhao, Z., L. Fang, N. Chen, R.C. Johnsen, L. Stein, and D.L. Baillie. 2005. Distinct regulatory elements mediate similar expression patterns in the excretory cell of Caenorhabditis elegans. The Journal of biological chemistry. 280:38787-38794.

Zheng, Y., D.J. Fischer, M.F. Santos, G. Tigyi, N.G. Pasteris, J.L. Gorski, and Y. Xu. 1996. The faciogenital dysplasia gene product FGD1 functions as a Cdc42Hsspecific guanine-nucleotide exchange factor. The Journal of biological chemistry. 271:33169-33172.

Zilberman, Y., J. Abrams, D.C. Anderson, and J. Nance. 2017. Cdc42 regulates junctional actin but not cell polarization in the Caenorhabditis elegans epidermis. The Journal of cell biology. 216:3729-3744. 
Zuo, X., B. Fogelgren, and J.H. Lipschutz. 2011. The small GTPase Cdc42 is necessary for primary ciliogenesis in renal tubular epithelial cells. The Journal of biological chemistry. 286:22469-22477.

Zuo, X., W. Guo, and J.H. Lipschutz. 2009. The exocyst protein Sec10 is necessary for primary ciliogenesis and cystogenesis in vitro. Molecular biology of the cell. 20:2522-2529. 
Figure 1
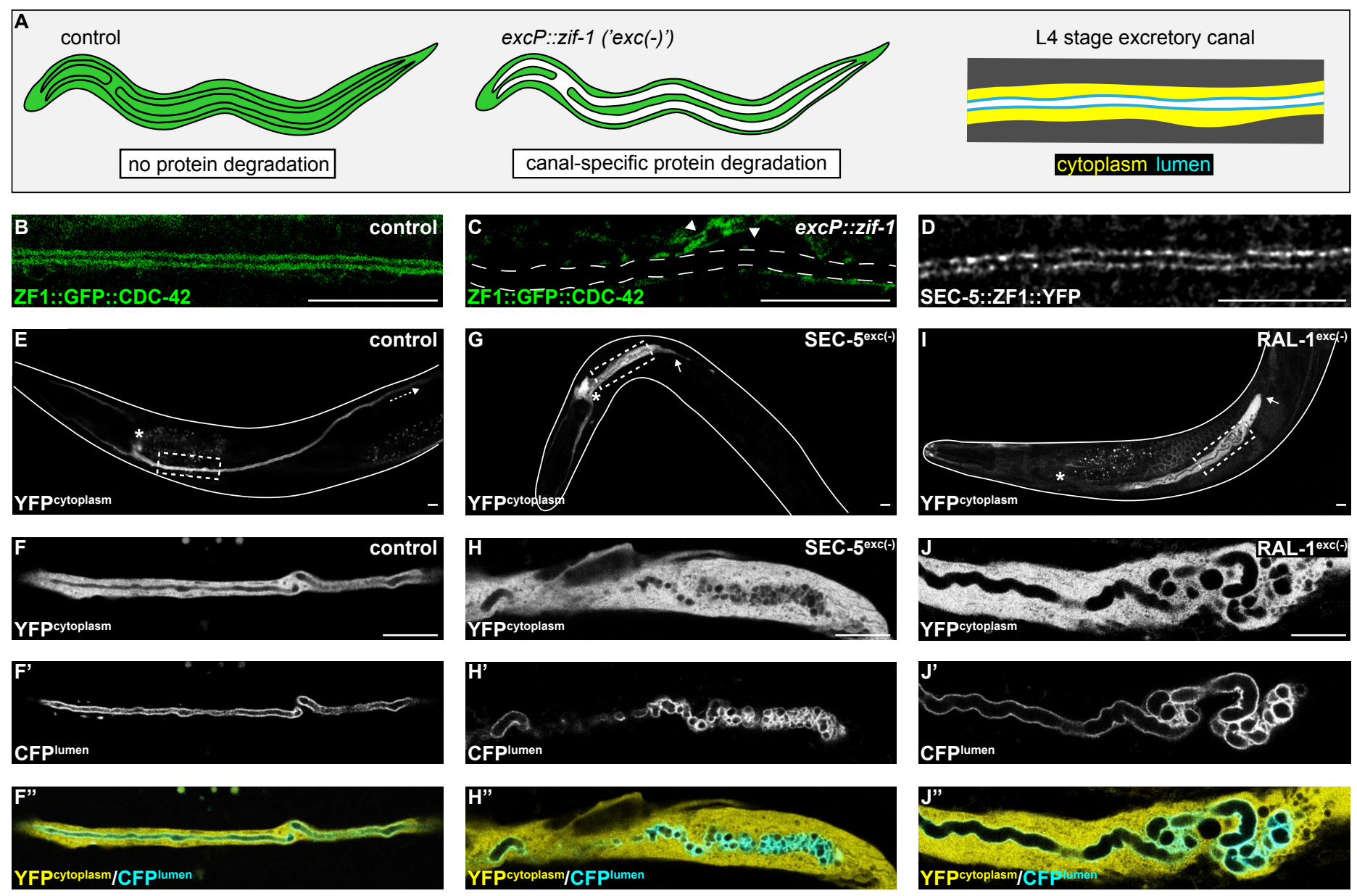
bioRxiv preprint doi: https://doi.org/10.1101/2020.10.05.327247; this version posted October 5, 2020. The copyright holder for this preprint (which was not certified by peer review) is the author/funder. All rights reserved. No reuse allowed without permission.

\section{Figure 2}

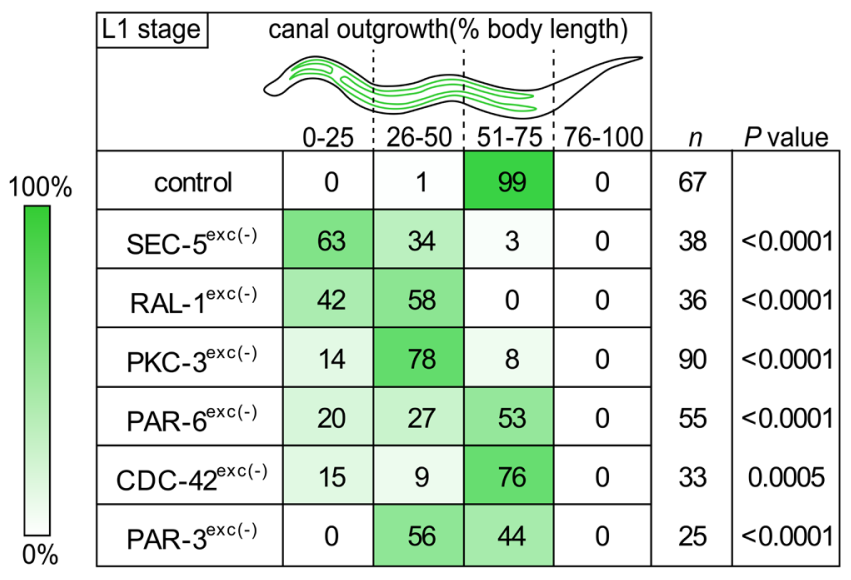

\begin{tabular}{|c|c|c|c|c|c|c|}
\hline \multirow[t]{2}{*}{\begin{tabular}{|l|} 
L4 stage \\
\end{tabular}} & al ou & owth( & body I & ngth) & \multirow[b]{2}{*}{$n$} & \multirow[b]{2}{*}{$P$ value } \\
\hline & $0-25$ & $26-50$ & $51-75$ & $76-100$ & & \\
\hline control & 0 & 0 & 2 & 98 & 108 & \\
\hline $\mathrm{SEC}-5^{\operatorname{exc}(-)}$ & 68 & 32 & 0 & 0 & 80 & $<0.0001$ \\
\hline RAL-1 $1^{\operatorname{exc}(-)}$ & 14 & 64 & 4 & 18 & 56 & $<0.0001$ \\
\hline$P K C-3^{\operatorname{exc}(-)}$ & 4 & 11 & 72 & 13 & 113 & $<0.0001$ \\
\hline PAR-6 $\operatorname{exc}^{\operatorname{ex}}$ & 20 & 15 & 15 & 50 & 117 & $<0.0001$ \\
\hline CDC $-42^{\operatorname{exc}(-)}$ & 6 & 16 & 23 & 55 & 144 & $<0.0001$ \\
\hline PAR-3 ${ }^{\operatorname{exc}(-)}$ & 0 & 0 & 2 & 98 & 62 & 0.4636 \\
\hline
\end{tabular}


Figure 3
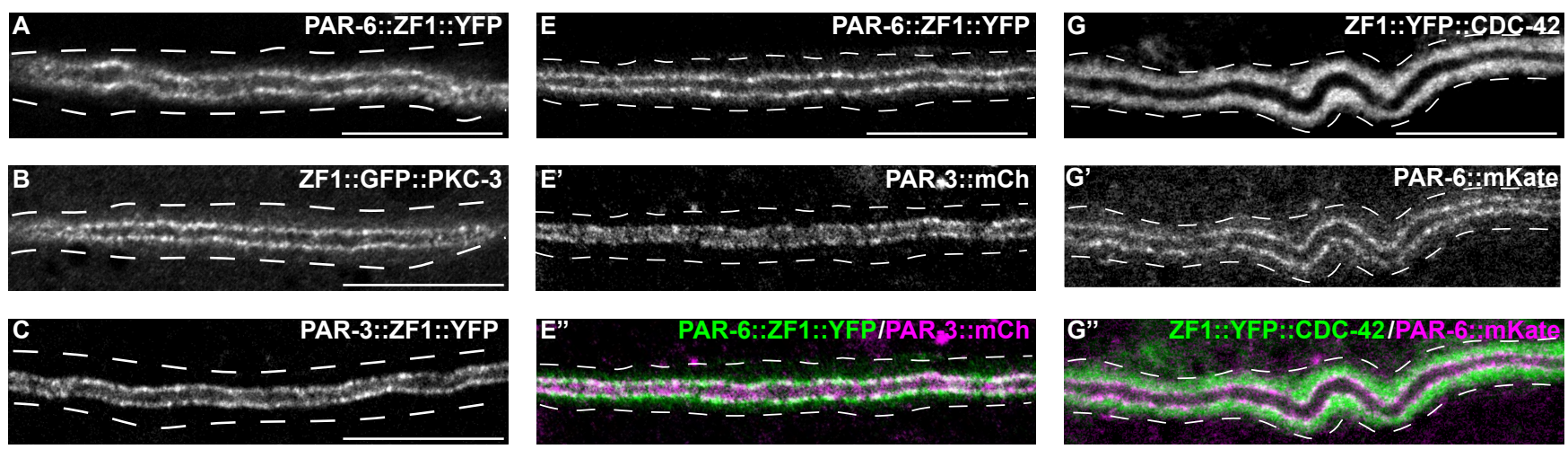

D

L4 stage excretory canal

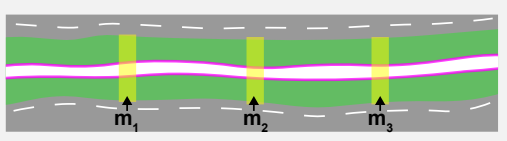

intensity plots $=$ average $\left(\mathrm{m}_{1}, \mathrm{~m}_{2}, \mathrm{~m}_{3}\right)$ $x 5$ worms
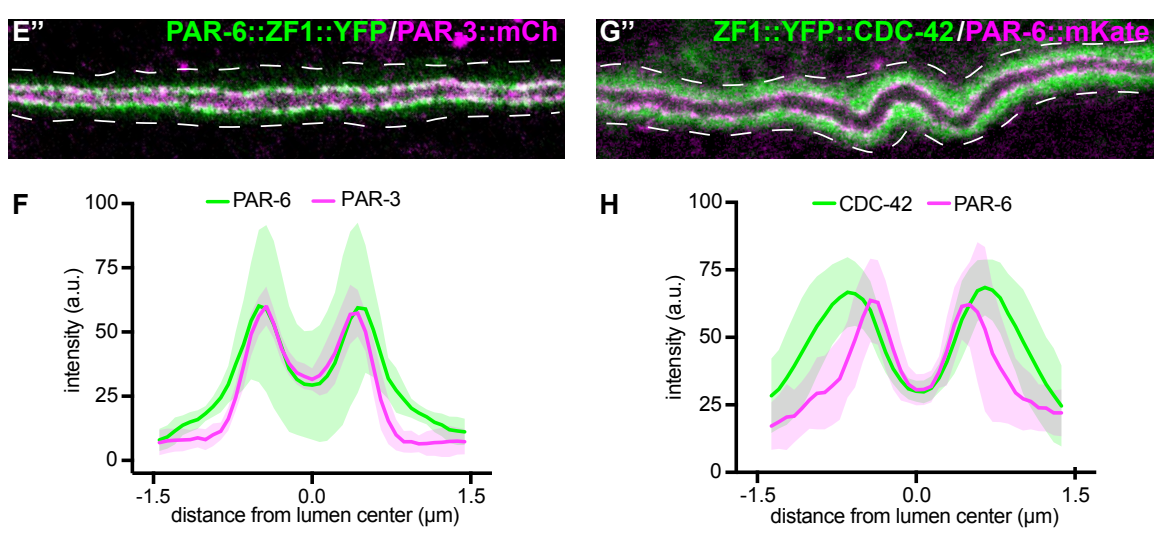
Figure 4
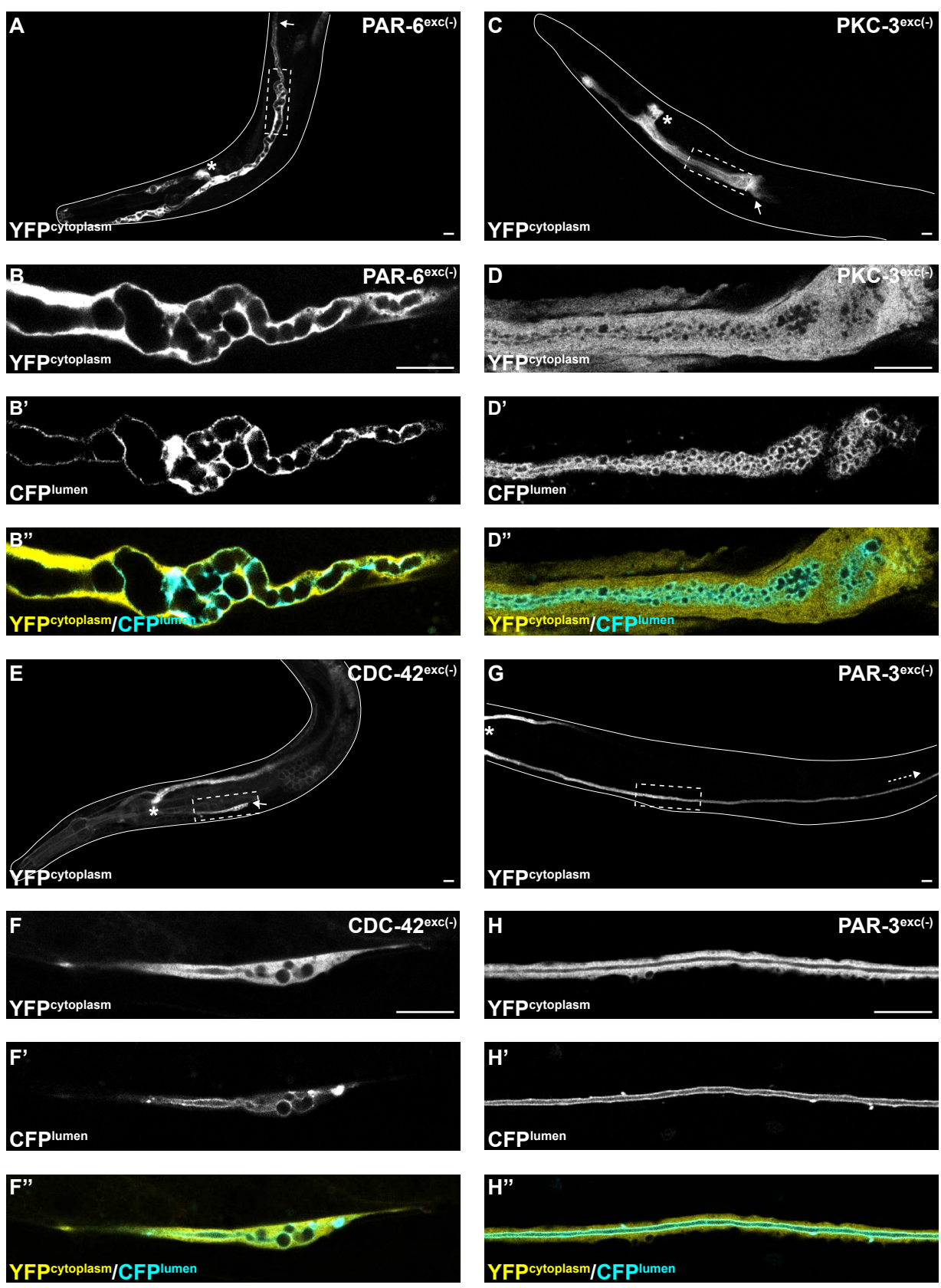


\section{Figure 5}
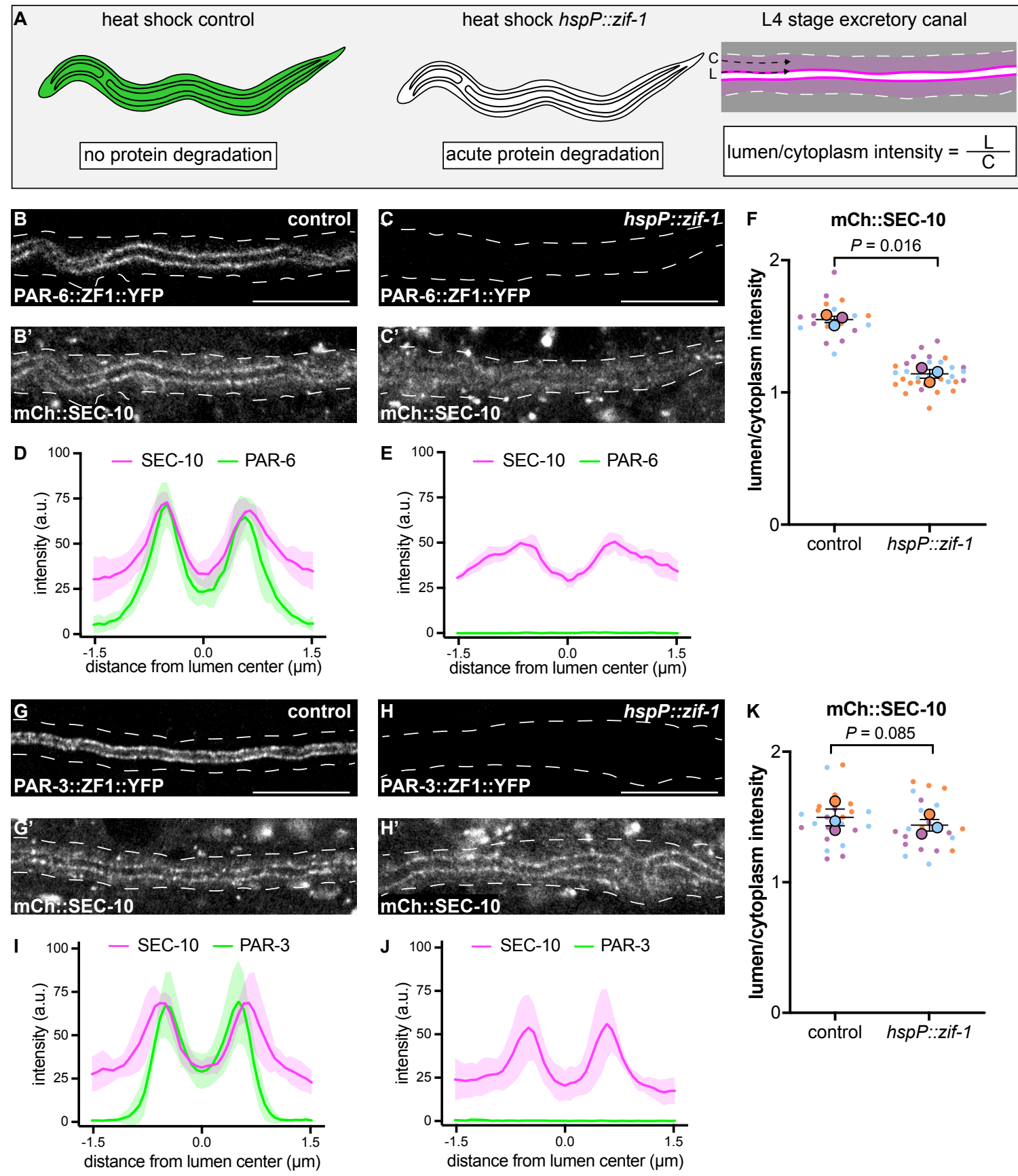


\section{Figure 6}
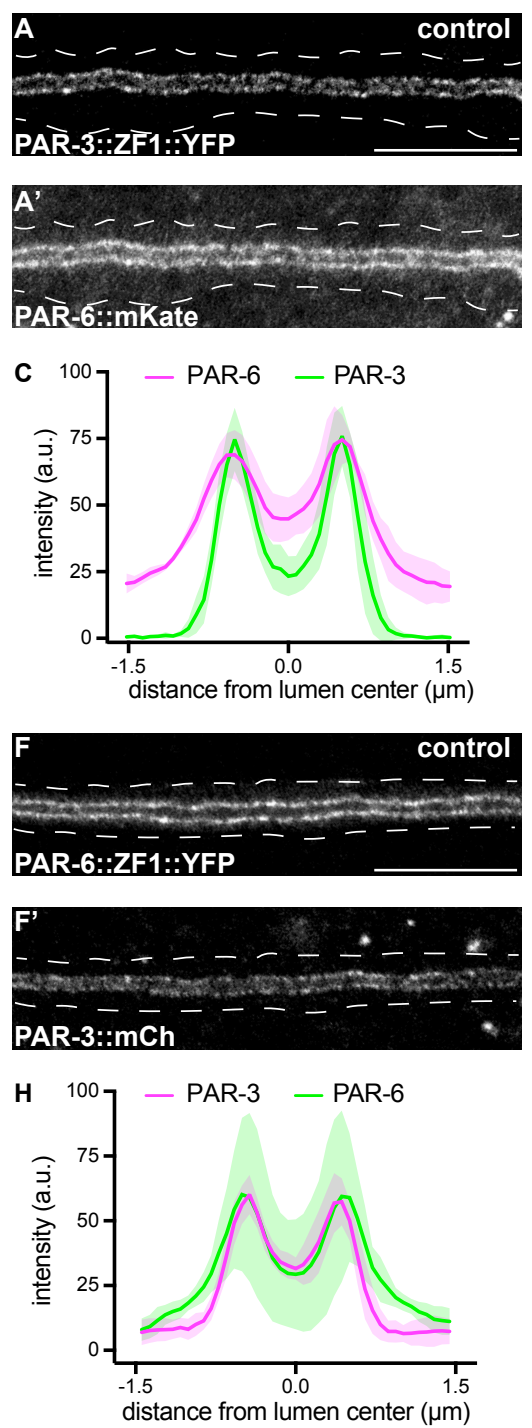
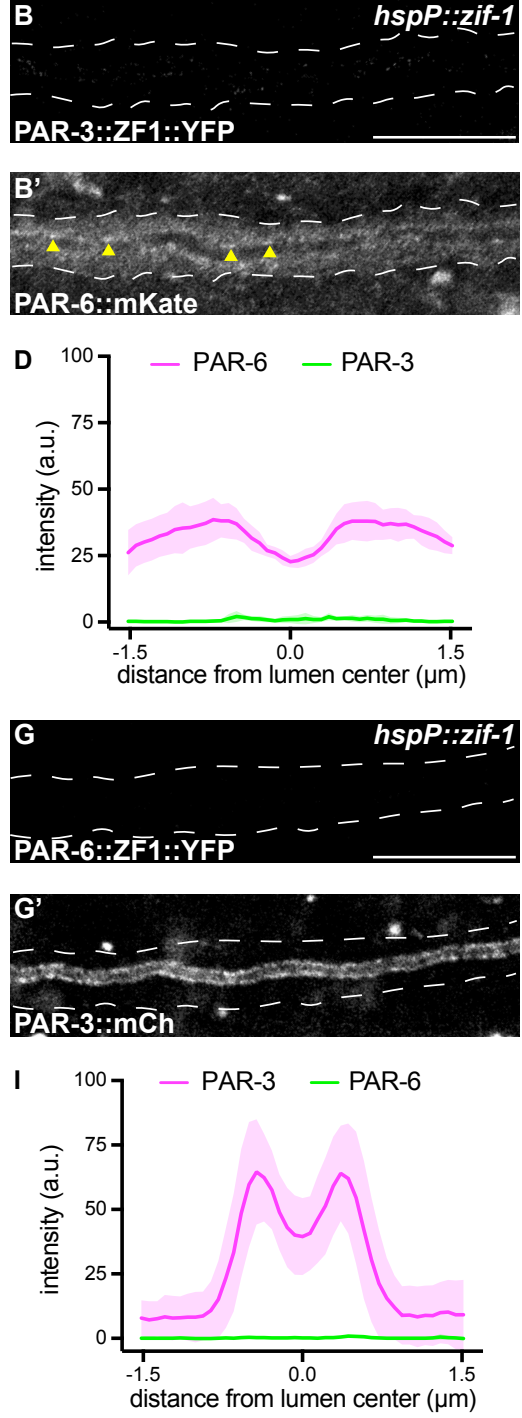
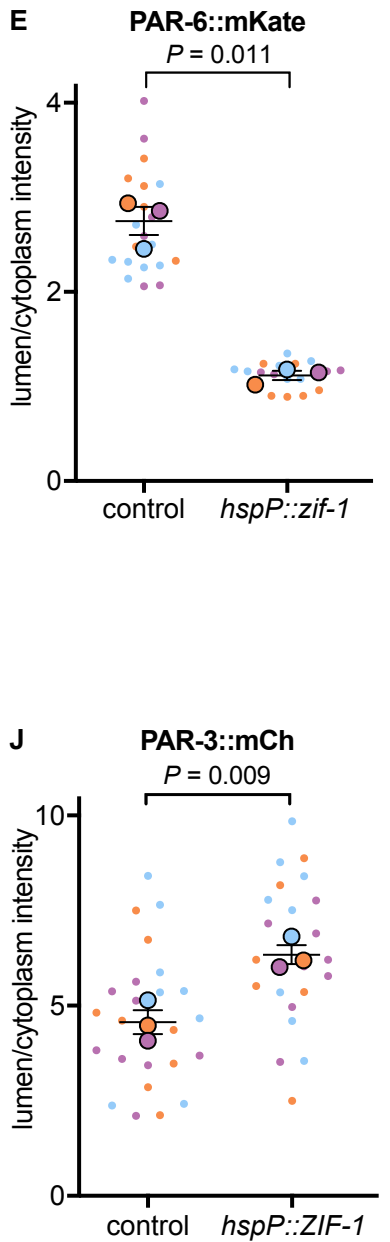
Figure 7
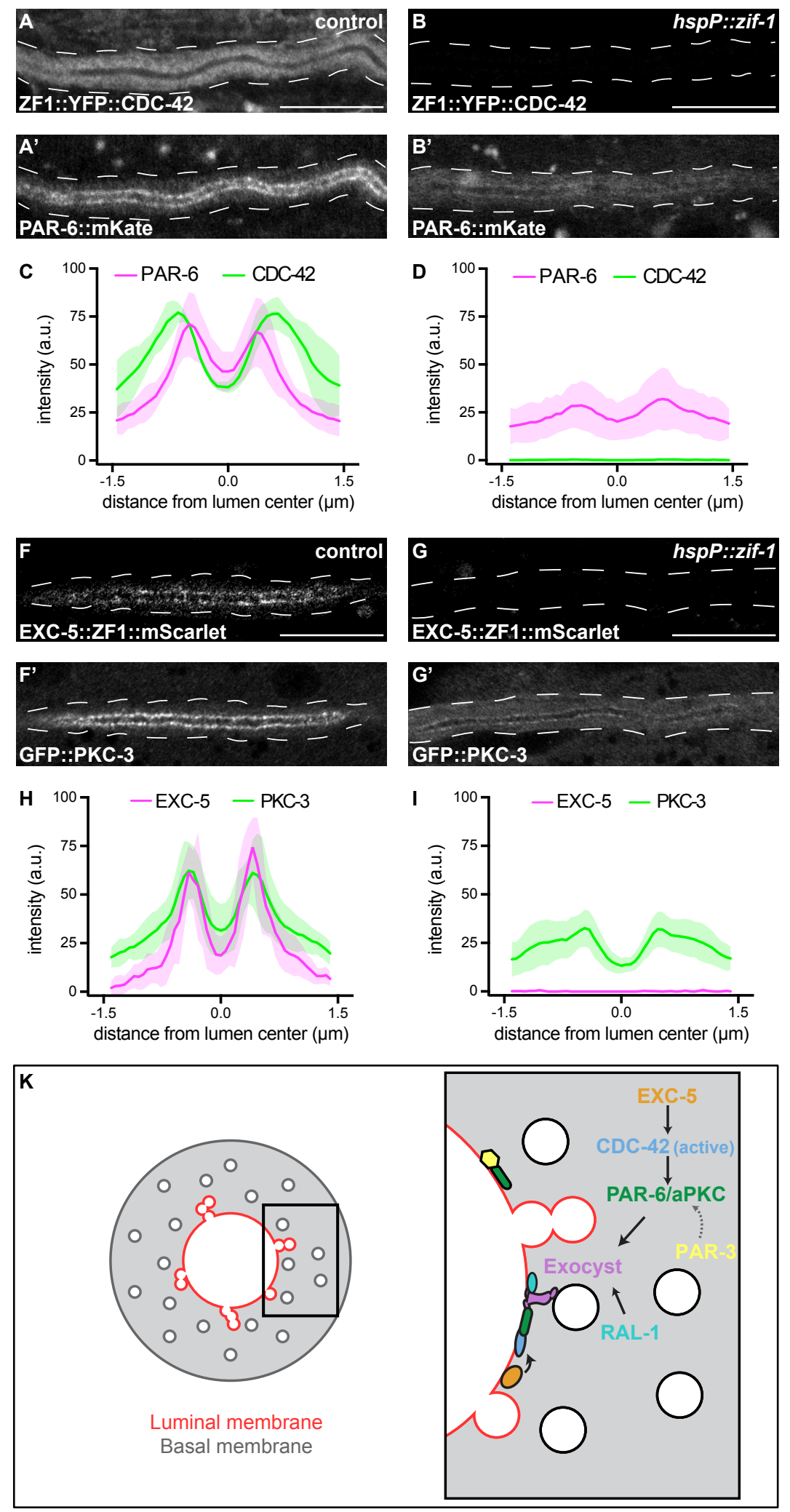
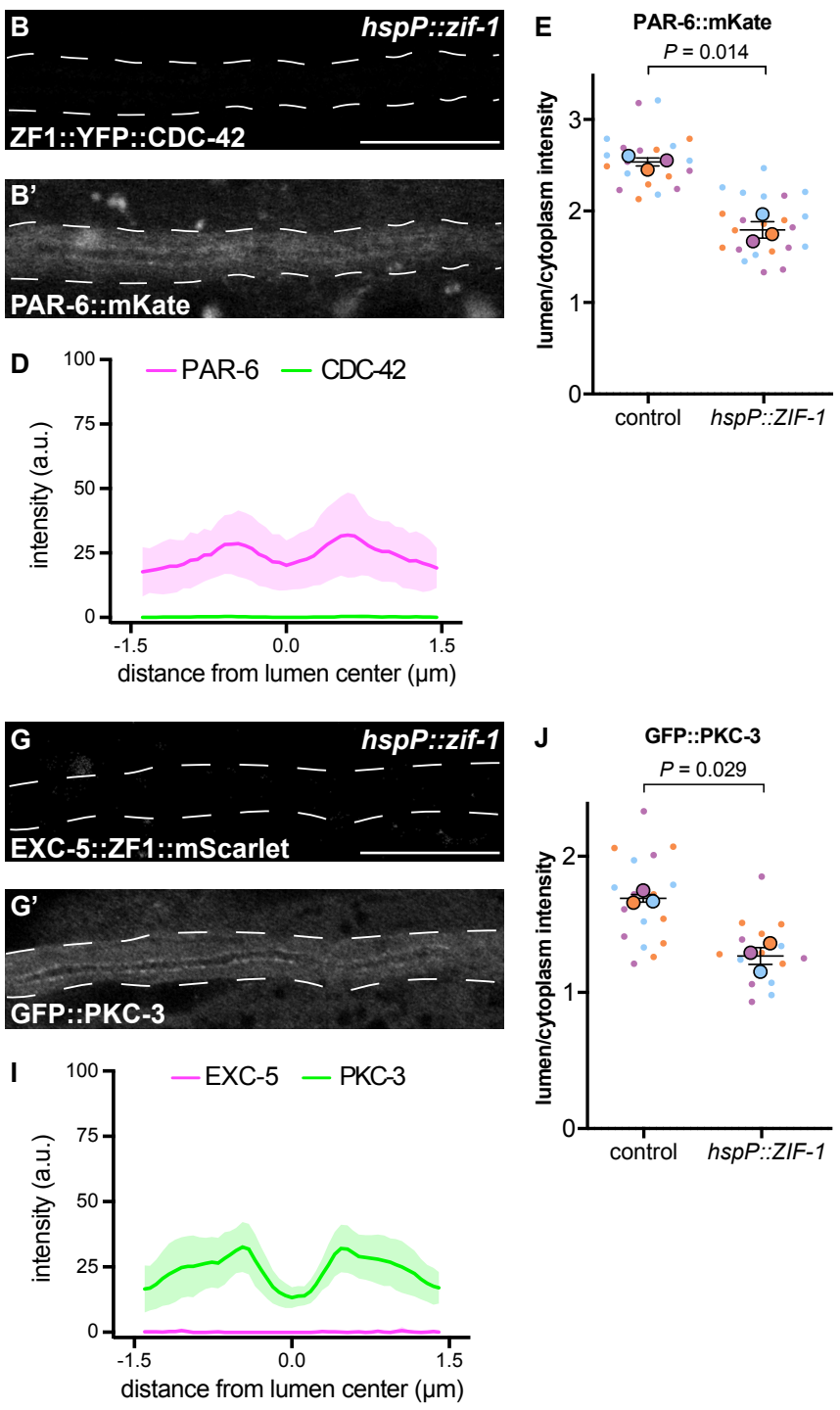